\title{
23. MAJOR-ELEMENT CHEMISTRY OF ARGILLACEOUS SEDIMENTS AT DEEP SEA DRILLING PROJECT SITES 442, 443, AND 444, SHIKOKU BASIN
}

\author{
Ryuichi Sugisaki, Department of Earth Sciences, Nagoya University, Nagoya, Japan
}

\begin{abstract}
Bulk chemical composition of 279 samples of argillaceous sediments from DSDP Sites 442, 443, and 444 in the Shikoku Basin (Leg 58) was determined and interpreted. Chemical features indicate that the Shikoku Basin sediments are typically terrigenous and were derived mostly from the Japanese Islands. A local contribution from oceanic islands, such as the Izu-Mariana Islands, to the basin sediments is not as large as expected from geophysical evidence. The Nankai Trough cannot be a barrier against sediment influx to the basin from the Japanese Islands. Except for $\mathrm{Ca}$ and $\mathrm{Mn}$, the bulk chemical composition of the sediments is inherited from the source materials, such as granites and volcanics in the Japanese Islands, without remarkable modification. Basal sediments immediately on the basaltic basement, enriched in $\mathrm{Mn}$ and $\mathrm{Fe}$, formed under the effect of hydrothermal emanations related to volcanism. From the chemical composition of the sediments, using the formula of Boström et al. (1976), the spreading rate of the Shikoku Basin at 17 to $15 \mathrm{Ma}$ is estimated to have been $1.7 \mathrm{~cm} / \mathrm{yr}$, which is not inconsistent with the rate determined from geophysical data.
\end{abstract}

\section{INTRODUCTION}

The Shikoku Basin is one of the most typical marginal basins, and it may provide the evidence to evaluate hypotheses for the genesis of the marginal basins of the western Pacific. The character of the sediments in the basin should constitute such evidence. Bulk chemical composition of 279 samples from DSDP Leg 58 Sites 442,443 , and 444 was determined and examined in this context.

Sugisaki (in press b) has studied sediments of the Japan Trench cored during DSDP Legs 56 and 57, and the chemical aspects of argillaceous sediments from piston cores around the Japanese Islands have been studied during cruises of the Geological Survey of Japan (Sugisaki, 1978; Sugisaki, 1979, in press a; Sugisaki and Honza, in press; Sugisaki and Kinoshita, in press).

The present paper gives critical comparisons of these data and discusses the distribution of elements in argillaceous sediments of the marginal basin, to examine possible sources of the deposits.

\section{ANALYTICAL PROCEDURE AND RESULT}

The analytical procedure adopted in this study is the same as that applied to the sediments of Legs 56 and 57 by Sugisaki (in press b).

The distribution of the samples and the analytical results are shown in Tables 1 through 5. Tables 6 through 10 list the silicate compositions of samples recalculated by excluding carbonate, salt, water, and residual materials (organic matter and others); these values are mostly used in the following discussion.

\section{GENERAL CHEMICAL CHARACTERISTICS OF THE SEDIMENTS}

$\mathrm{CaCO}_{3}$ was found in some of the samples and was not detected in others (Tables 1 through 5). This suggests that the depositional surface fluctuated above or below the calcium carbonate compensation depth (CCD) during the depositional history. Few data are available concerning present or past level of the CCD in the Shikoku Basin, and the exact depth of deposition cannot be determined. Sugisaki (1978) inferred from piston core data that the CCD in the Nankai Trough and its vicinity is at about 4500 meters. Present water depths at Sites 442,443 , and 444 are 4639,4372 and 4843 meters, respectively. $\mathrm{CaCO}_{3}$ was not found in the upper part of the cores at Site 442 , but it was detected in the others. This evidence is not inconsistent with that for the Nankai Trough.

Table 11 lists the average silicate composition of the sediments at each site. They are closely similar. Furthermore, almost all of the standard deviations are small, which shows a high degree of uniformity of the argillaceous sediments through the entire sampled sequences.

For comparison, several other sets of averages are shown in Table 11, representing piston-core sediments from the Philippine Sea far south of the Leg 58 area, pelagic sediments from the east Pacific Ocean, and argillaceous sediments from five regions around the Japanese Islands. The samples from the Oki area and the Japan Trench are contaminated by quartz (Sugisaki and Kinoshita, in press) and biogenic silica (Sugisaki, in 
TABLE

Chemical Composition (\%) of Argillaceous Sediments of Hole 442A

\begin{tabular}{|c|c|c|c|c|c|c|c|c|c|c|c|c|c|c|c|c|c|}
\hline No. & $\begin{array}{c}\text { Sample } \\
\text { (interval in } \mathrm{cm} \text { ) }\end{array}$ & $\mathrm{SiO}_{2}$ & $\mathrm{TiO}_{2}$ & $\mathrm{Al}_{2} \mathrm{O}_{3}$ & $\mathrm{Fe}_{2} \mathrm{O}_{3}$ & $\mathrm{FeO}$ & $\mathrm{MnO}$ & $\mathrm{MgO}$ & $\mathrm{CaO}$ & $\mathrm{Na}_{2} \mathrm{O}$ & $\mathrm{K}_{2} \mathrm{O}$ & $\mathrm{P}_{2} \mathrm{O}_{5}$ & $\mathrm{H}_{2} \mathrm{O}$ & $\mathrm{CaCO}_{3}$ & $\begin{array}{l}\text { Resid- } \\
\text { ual* }\end{array}$ & Salt & Total \\
\hline 1 & $442 \mathrm{~A}-2-1,91-93$ & 59.96 & 0.68 & 16.00 & 3.58 & 2.30 & 0.10 & 2.45 & 1.34 & 1.30 & 2.96 & 0.13 & 6.51 & 0.0 & 1.55 & 2.15 & 101.00 \\
\hline 2 & $2-2$ & 60.38 & 0.67 & 15.74 & 3.73 & 2.01 & 0.11 & 2.25 & 1.51 & 1.40 & 2.85 & 0.14 & 5.30 & 0.0 & 0.94 & 2.06 & 99.09 \\
\hline 3 & $2-3,60-62$ & 58.36 & 0.66 & 15.46 & 3.62 & 2.27 & 0.12 & 2.18 & 1.45 & 1.25 & 2.94 & 0.13 & 6.59 & 0.0 & 1.67 & 2.26 & 98.95 \\
\hline 4 & $2-4,71-73$ & 57.20 & 0.68 & 15.79 & 3.26 & 2.33 & 0.15 & 2.29 & 0.84 & 0.93 & 3.21 & 0.11 & 6.86 & 3.12 & 1.53 & 1.92 & 100.22 \\
\hline 5 & $2-5,13-15$ & 64.34 & 0.55 & 15.49 & 2.69 & 1.90 & 0.10 & 1.82 & 1.25 & 1.20 & 3.11 & 0.11 & 6.47 & 0.0 & 0.09 & 1.85 & 100.97 \\
\hline 6 & $2-6,36-38$ & 60.39 & 0.67 & 16.02 & 3.52 & 2.31 & 0.12 & 2.46 & 1.45 & 0.94 & 3.14 & 0.11 & 5.67 & 0.0 & 0.39 & 2.07 & 99.27 \\
\hline 7 & $3-2,30-32$ & 60.75 & 0.58 & 14.97 & 2.95 & 2.02 & 0.24 & 2.03 & 2.35 & 1.31 & 3.13 & 0.09 & 5.70 & 0.0 & 1.41 & 1.72 & 99.27 \\
\hline 8 & $3-3,46-48$ & 57.52 & 0.65 & 15.34 & 3.30 & 2.11 & 0.28 & 2.53 & 0.91 & 1.13 & 3.13 & 0.11 & 5.01 & 3.73 & 1.23 & 2.00 & 98.98 \\
\hline 9 & $3-4,53-55$ & 58.98 & 0.70 & 16.34 & 3.30 & 2.38 & 0.13 & 2.26 & 1.20 & 0.98 & 3.20 & 0.11 & 5.36 & 0.0 & 2.35 & 1.75 & 99.05 \\
\hline 10 & $3-5,100-102$ & 59.62 & 0.70 & 16.33 & 3.23 & 2.46 & 0.21 & 2.46 & 1.41 & 1.26 & 3.23 & 0.12 & 6.51 & 0.0 & 1.49 & 1.64 & 100.67 \\
\hline 11 & $3-6,127-129$ & 55.97 & 0.69 & 16.36 & 3.53 & 2.24 & 0.15 & 2.46 & 0.07 & 1.26 & 3.22 & 0.10 & 7.41 & 4.33 & 0.94 & 1.84 & 100.57 \\
\hline 12 & $3-7,68-70$ & 57.95 & 0.68 & 15.43 & 3.50 & 2.19 & 0.27 & 2.46 & 0.57 & 1.42 & 2.04 & 0.13 & & 4.91 & & 1.72 & 100.14 \\
\hline 13 & $41,63-65$ & 56.67 & 0.67 & 15.82 & 3.66 & 2.22 & 0.17 & 2.37 & 1.68 & 0.94 & 3.12 & 0.11 & 7.55 & 0.0 & 0.45 & 3.59 & 99.03 \\
\hline 14 & $4-2,105-107$ & 59.32 & 0.70 & 15.97 & 3.93 & 2.17 & 0.17 & 2.52 & 1.17 & 1.18 & 3.23 & 0.12 & 6.41 & 0.0 & 0.17 & 2.22 & 99.28 \\
\hline 15 & $4-3,20-22$ & 53.92 & 0.65 & 14.73 & 3.40 & 2.17 & 0.35 & 2.39 & 0.62 & 1.18 & 3.01 & 0.13 & 6.54 & 7.73 & 0.18 & 2.04 & 99.02 \\
\hline 16 & $4-4,35-37$ & 52.50 & 0.64 & 14.39 & 3.56 & 2.00 & 0.20 & 2.30 & 0.55 & 0.42 & 3.05 & 0.11 & 5.75 & 11.03 & 0.20 & 2.34 & 99.04 \\
\hline 17 & $4-5,7-9$ & 56.47 & 0.67 & 15.57 & 3.28 & 2.14 & 0.20 & 2.23 & 0.31 & 1. & 3.17 & & & 5.77 & 9 & 1.97 & 99.04 \\
\hline 18 & $5-1,111-113$ & 60.36 & 0.68 & 17.04 & 3.50 & 2.15 & 0.23 & 2.44 & 1.31 & 1.37 & 3.11 & 0.13 & 5.97 & 0.0 & 0.18 & 2.17 & 100.65 \\
\hline 19 & $5-2,106-108$ & 56.99 & 0.69 & 15.88 & 3.96 & 2.08 & 0.23 & 2.45 & 1.25 & 1.28 & 3.25 & 0.11 & 7.05 & 0.0 & 2.07 & 1.92 & 99.21 \\
\hline 20 & 5 & 60.27 & 0.66 & 15.75 & 3.68 & 2.05 & 0.37 & 2.30 & 0.14 & 1.49 & 3.26 & 0.10 & 5.32 & 3.33 & 0.16 & 1.95 & 100.83 \\
\hline 21 & $5-4,131-133$ & 57.75 & 0.73 & 16.37 & 3.48 & 2.26 & 0.63 & 2.66 & 1.10 & 1.79 & 3.28 & 0.11 & 5.17 & 0.0 & 1.93 & 1.74 & 99.00 \\
\hline 22 & $5,118-120$ & 60.67 & 0.74 & 17.39 & 3.22 & 2.33 & 0.18 & 2.64 & 0.14 & 1. & 3. & 0. & & & & 1.48 & 101.01 \\
\hline 23 & $6-1,121-123$ & 59.17 & 0.75 & 16.38 & 3.61 & 2.34 & 0.19 & 2.56 & 1.02 & $1 .($ & 3.33 & 0.11 & 6.44 & 0.0 & 0.11 & 2.04 & 99.07 \\
\hline 24 & $2,10-12$ & 58.38 & 0.66 & 15.26 & 3.32 & 2.12 & 0.43 & 2.36 & 0.09 & 1.44 & 3.28 & 0.11 & 5.96 & 3.63 & 0.66 & 1.48 & 99.19 \\
\hline 25 & $8-110$ & 60.25 & 0.66 & 15.99 & 3.54 & 2.06 & 0.43 & 2.52 & 1.36 & 1.75 & 3.21 & 0.17 & 6.91 & 0.0 & 0.08 & 2.13 & 101.05 \\
\hline 26 & $4,45-47$ & 60.58 & 0.71 & 16.80 & 3.72 & 2.18 & 0.25 & 2.62 & 0.35 & 1.55 & 3.37 & 0.11 & 5.48 & 1.50 & 0.19 & 1.52 & 100.93 \\
\hline 27 & 12 & 60.66 & 0.69 & 17.09 & 3.72 & 2.00 & 0.27 & 2. & 1.19 & 1. & 3. & 0.13 & & 0 . & & 1.47 & 101.14 \\
\hline 28 & $-6,5-7$ & 58.30 & 0.67 & 15.60 & 3.61 & 2.12 & 0.31 & 2.36 & 1.25 & 1.5 & 3.12 & 0.11 & 6. & 0 . & 1. & 1.61 & 99.06 \\
\hline 29 & $7-1,129-131$ & 57.57 & 0.70 & 16.18 & 4.23 & 2.02 & 0.45 & 2.67 & 0.94 & 1.8 & & 0.12 & 7.28 & 0. & 0.32 & 1.43 & 99.05 \\
\hline 30 & $7-2,34-36$ & 59.61 & 0.70 & 16.84 & 3.41 & 2.22 & 0.38 & 2.71 & 0.02 & 2.15 & 3.27 & 0.12 & 6.82 & 0.0 & 0.47 & 1.51 & 100.23 \\
\hline 31 & $7-3,34-36$ & 59.38 & 0.71 & 16.79 & 4.33 & 2.04 & 0,49 & 2.84 & 1.09 & 2.03 & 3.18 & 0.12 & 6.07 & 0.0 & 0.35 & 1.56 & 100.97 \\
\hline 32 & $4,34-36$ & 63.86 & 0.48 & 14.40 & 2.41 & 1.60 & 0 . & 1.67 & 1.10 & 1.6 & 3.24 & 0.08 & 5. & & 5 & 5 & 99.19 \\
\hline 33 & $7-5,34-36$ & 58.43 & 0.68 & 16.49 & 3.62 & 2.20 & 0.36 & 2.69 & 0.40 & 1.63 & 3. & 0.11 & 6. & 3.47 & & 1.30 & 100.88 \\
\hline 34 & $8-4,45-47$ & 58.85 & 0.69 & 17.05 & 3.92 & 2.08 & 0.41 & 2.69 & 1.17 & 1.5 & 3.19 & 0.1 & & 0. & & 1.52 & 100.49 \\
\hline 35 & $8-5,45-47$ & 61.71 & 0.67 & 16.11 & 4.02 & 1.97 & 0.37 & 2.58 & 1.28 & 1.76 & 3.08 & 0.12 & 5.97 & 0.0 & 0.14 & 1.23 & 101.00 \\
\hline 36 & $10-3,11-13$ & 56.70 & 0.70 & 16.69 & 4.37 & 2.13 & 0.41 & 2.92 & 1.11 & 1.79 & 3.24 & 0.12 & 5.65 & 0.0 & 1.72 & 1.57 & 99.12 \\
\hline 37 & $10-4,1$ & 58.29 & 0.59 & 14.53 & 5 & 1.67 & 0. & 2 & 0.22 & 2.42 & 3.33 & 0. & 6. & 1. & 0. & 1.52 & 99.80 \\
\hline 38 & $11-3,6-8$ & 57.97 & 0.73 & 17.48 & 3.71 & 2.24 & 0.25 & 2.83 & 1.01 & 1.51 & 3.51 & 0 . & & 0 . & & 1.53 & 100. \\
\hline 39 & $13-2,100-102$ & 51.66 & 0.67 & 15.86 & 3.39 & 2.37 & 2.70 & 3.47 & 0.83 & 4.75 & 2.84 & 1. & & & 0.72 & 1.37 & 101.40 \\
\hline 40 & $14-3,99-101$ & 56.21 & 0.75 & 17.28 & 4.53 & 1.96 & 0.29 & 2.66 & 1.07 & 1.50 & 3.13 & 0.12 & 7.42 & 0.0 & 0.90 & 1.26 & 99.08 \\
\hline 41 & $15-2,69-71$ & 54.37 & 0.70 & 16.44 & 4.76 & 1.61 & 2.01 & 3.51 & 0.10 & 3.87 & 3.01 & 0.12 & 6.23 & 2.01 & 0.04 & 1.64 & 100.42 \\
\hline 42 & $-3,44-46$ & 56.94 & 0.72 & 17.57 & 4 & 1.72 & 0.12 & 2.63 & 1.04 & 1.9 & 3.0 & 0. & 7. & 0. & 0.77 & 0.77 & 98. \\
\hline 43 & $15-4,9-11$ & 57.40 & 0.74 & 17.18 & 5.44 & 1.53 & 0.24 & 2.34 & 1.21 & 1.26 & 2.92 & & 6.47 & 0 & & & 99. \\
\hline 44 & $16-1,93-95$ & 56.97 & 0.73 & 17.53 & 4.72 & 1.64 & 0.11 & 2.33 & 0.87 & 1.12 & 3.17 & 0.12 & 7.38 & 0 . & & 1.73 & 99.11 \\
\hline 45 & $16-2,33-35$ & 56.81 & 0.72 & 17.22 & 4.45 & 1.78 & 0.42 & 2.69 & 0.98 & 1.40 & 3.29 & 0.12 & 6.07 & 0.0 & 1.32 & 1.79 & 99.05 \\
\hline 46 & $17-2,84-86$ & 59.07 & 0.70 & 16.78 & 4.98 & 1.54 & 0.21 & 2.63 & 1.36 & 1.57 & 3.0 & 0.1 & 5.70 & 0. & 1.76 & 1.42 & 100.94 \\
\hline 47 & $18-2,135-137$ & 56.97 & 0.71 & 17.45 & 4.95 & 1.71 & 0.36 & 2.73 & 1.66 & 1.6 & 2.98 & & & 0 . & & 89 & 100. \\
\hline 48 & $18-3,7-9$ & 56.16 & 0.74 & 16.70 & 5.13 & 1.42 & 0.90 & 2.59 & 1.54 & & & & & & & & \\
\hline 49 & $19-1,130-132$ & 56.24 & 0.74 & 17.81 & 5.38 & 1.53 & 0.35 & 2.81 & 0.12 & 1.72 & 3.12 & 0.13 & 5.60 & 2.19 & 0.65 & 1.36 & \\
\hline 50 & $19-2,60-62$ & 57.12 & 0.73 & 17.73 & 5.31 & 1.51 & 0.51 & 2.79 & 0.55 & 1.80 & 3.17 & 0.15 & 6.36 & 1.46 & 0.05 & 1.51 & 100.75 \\
\hline 51 & $-3,88-90$ & 57.80 & 0.73 & 17.64 & 5,43 & 1.51 & 0.44 & 2.89 & 0.22 & 1.48 & 3.09 & 0.1 & 5.80 & 1.8 & 0.19 & 1.69 & 100.91 \\
\hline 52 & $20-1,100-102$ & 55.86 & 0.71 & 17.58 & 5.40 & 1.38 & 0.72 & 2.80 & & & & & & & & 1.61 & 100. \\
\hline 53 & $21-1,16-18$ & 57.62 & 0.69 & 17.35 & 5.40 & 1.41 & 0.20 & 2.39 & 1.14 & 1.17 & 3.16 & & 7.13 & 0.0 & & 1.21 & \\
\hline 54 & $-2,16-18$ & 58.27 & 0.72 & 17.93 & 5.57 & 1.46 & 0.13 & 2.66 & 1.18 & & 3.18 & 0 . & 6.59 & 0 & 0.58 & 1.14 & 100.95 \\
\hline 55 & $21-3,16-18$ & 60.05 & 0.69 & 17.80 & 5.45 & 1.39 & 0.17 & 2.56 & 1.21 & 1. & 3.00 & 0.17 & 6.01 & 0. & 0.15 & 1.09 & 100.96 \\
\hline 56 & 4.16 & 57.26 & 0.69 & 17.1 & 6.27 & 0.49 & 0.72 & 2.43 & 0.93 & 1.75 & 3.1 & 0 . & 6. & 0 & 0.37 & 1.09 & 99.28 \\
\hline 57 & $-4,20-22$ & 58.06 & 0.70 & 17.66 & & & & & & & & & & & & 47 & 92 \\
\hline 58 & $-1, C C$ & 57.77 & 0.70 & 17.50 & 5 & 1.07 & 0.31 & & & & & & & & 0.17 & 1.12 & \\
\hline 59 & $-1,28-30$ & 55.54 & 0.70 & 17.54 & 4 & 1 & 0. & 3 & & 2 & 3. & 0 . & 6. & 2. & 0 & 1.09 & 100. \\
\hline 60 & $23-3,57-59$ & 56.01 & 0.71 & 17.16 & 5.37 & 1.29 & 1.02 & 3.20 & & & & 0.1 & & 2. & 0.07 & 1.14 & 100.9 \\
\hline 61 & $23-4,143-145$ & 59.50 & 0.71 & 18.16 & 5.35 & 1.49 & 0.13 & 2.43 & 1.18 & 1.36 & 3.06 & 0.13 & 6.30 & 0. & 0.38 & 1.12 & 101.29 \\
\hline 62 & 5 & 58.40 & 2 & 81 & & & & & & & & & & & & & \\
\hline 63 & $1,128-130$ & 58.42 & 0.66 & 16.67 & .01 & 3 & 0. & & & 2 & 3. & 0. & & 0. & 0 & 3 & 100. \\
\hline 64 & $24-2,140-142$ & 58.11 & 0.67 & 16.55 & 5.11 & 1.11 & 0. & 2. & 0. & 2. & & & & & & & 100.86 \\
\hline 65 & $24-3,50-52$ & 58.75 & 0.66 & 17.55 & 5.51 & 0.81 & 0.4 & 2.47 & 1.72 & 2. & 2.8 & 0. & 6. & 0 . & 0.17 & 1.19 & 100.98 \\
\hline 66 & $24-4,50-52$ & 57.64 & 0.66 & 16.68 & 5.29 & 1.20 & 0.58 & 2.55 & 1.78 & 2.23 & 2.92 & 0.18 & 7.15 & 0 . & 0.39 & 1.26 & 100.50 \\
\hline 67 & $5,50-52$ & 57.97 & 0 & & & & & & & & & & & 0 . & & 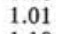 & 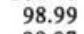 \\
\hline 68 & $-2,111-113$ & 57.00 & 0.6 & , & & & 0. & & & & & & & & & & \\
\hline 69 & $3,126-128$ & 57.08 & 0.69 & 16.69 & & 1.51 & 1.21 & & & & & & & & & 1.21 & \\
\hline 70 & , $126-128$ & 58.04 & 0.70 & 16.95 & 5.48 & 1.65 & 0.25 & 2.46 & 2.47 & 1.76 & 2.6 & 0.1 & 4.60 & 0.0 & 1.70 & 1.21 & \\
\hline 71 & $26-2,100-102$ & 57.06 & & 16.09 & 5.71 & 1.41 & 0.53 & 3.1 & 0.85 & 2.29 & 2.47 & 0.15 & 5.77 & 3.04 & 0.03 & & 100.47 \\
\hline 72 & $-2,124-126$ & 56.18 & & & & & & & & & & & & & 0 & 1.94 & . \\
\hline 73 & $28-1,62-64$ & 57.64 & 0.66 & 16.2 & & & 0.42 & & & & & & & & & 1.90 & 100. \\
\hline 74 & $28-2,53-55$ & 54.92 & 0.66 & 16.24 & & 1.19 & 0.44 & & & & & & & 0. & & & \\
\hline 75 & $29-1,88-90$ & 53.60 & 0.62 & 14.58 & & 0.16 & 0.28 & 3.75 & 2.22 & 2.09 & 1.87 & & 7.07 & 0.0 & & 1.80 & \\
\hline 76 & $29-$ & 53.42 & 0.63 & 15.05 & 7 & 0.56 & 0.74 & 3.61 & 2.68 & 1.71 & 2.28 & 0.23 & 8.06 & 0 . & 0.34 & 2.12 & 99.04 \\
\hline 77 & 51 & 53.57 & 0.67 & & & & & & 2.27 & & 2.31 & & & 0. & 0.34 & 0.92 & 99.3 \\
\hline 78 & $30-2,22-24$ & 54.56 & 0.64 & 15. & 8. & 0.05 & 1.2 & 4.29 & 1.75 & 2.90 & 3.01 & 0.28 & & 0. & & 0.78 & 100.93 \\
\hline 79 & $30-3,76-78$ & 51.01 & 0.60 & 14.39 & 9.41 & 0.04 & 1.67 & 4.29 & 1.62 & 0.52 & 3.05 & 0.51 & 7.16 & 0.0 & 0.21 & 4.61 & \\
\hline
\end{tabular}

*Residual materials were calculated by subtracting $\mathrm{CO}_{2}$ and $\mathrm{H}_{2} \mathrm{O}$ from ignition loss. They may contain sulfur, organic matter, and other materials. 
TABLE 2

Chemical Composition (\%) of Argillaceous Sediments of Hole 442B

\begin{tabular}{|c|c|c|c|c|c|c|c|c|c|c|c|c|c|c|c|c|c|}
\hline No. & $\begin{array}{c}\text { Sample } \\
\text { (interval in } \mathrm{cm} \text { ) }\end{array}$ & $\mathrm{SiO}_{2}$ & $\mathrm{TiO}_{2}$ & $\mathrm{Al}_{2} \mathrm{O}_{3}$ & $\mathrm{Fe}_{2} \mathrm{O}_{3}$ & $\mathrm{FeO}$ & $\mathrm{MnO}$ & $\mathrm{MgO}$ & $\mathrm{CaO}$ & $\mathrm{Na}_{2} \mathrm{O}$ & $\mathrm{K}_{2} \mathrm{O}$ & $\mathrm{P}_{2} \mathrm{O}_{5}$ & $\mathrm{H}_{2} \mathrm{O}$ & $\mathrm{CaCO}_{3}$ & Residual* & Salt & Total \\
\hline 1 & 442B-1-1, 76-78 & 68.85 & 0.35 & 12.71 & 1.39 & 0.77 & 0.061 & 0.69 & 1.67 & 1.96 & 3.96 & 0.11 & 4.02 & 0.0 & 1.23 & 1.26 & 99.03 \\
\hline 2 & $1-2,24-26$ & 55.66 & 0.65 & 15.72 & 5.56 & 1.39 & 0.026 & 2.83 & 2.16 & 1.14 & 2.64 & 0.16 & 3.23 & 0.0 & 5.56 & 2.42 & 99.14 \\
\hline 3 & $1-2,137-139$ & 66.36 & 0.46 & 13.21 & 2.34 & 1.29 & 0.41 & 1.03 & 2.22 & 3.05 & 3.33 & 0.13 & 4.92 & 0.0 & 1.12 & 1.09 & 100.96 \\
\hline 4 & $2-1,36-38$ & 47.43 & 0.57 & 12.88 & 8.51 & 0.03 & 1.16 & 3.70 & 1.96 & 3.05 & 1.98 & 0.32 & 6.38 & 9.70 & 0.71 & 1.79 & 100.16 \\
\hline 5 & $2-2,17-19$ & 50.86 & 0.59 & 13.44 & 9.75 & 0.05 & 1.99 & 4.98 & 1.81 & 4.45 & 2.34 & 0.35 & 7.18 & 0.0 & 1.71 & 1.61 & 101.11 \\
\hline 6 & $2-3,9-11$ & 51.11 & 0.58 & 13.87 & 8.58 & 0.12 & 1.58 & 5.21 & 1.89 & 3.67 & 2.29 & 0.31 & 8.70 & 0.0 & 0.77 & 1.80 & 100.47 \\
\hline 7 & $2-4,62-64$ & 52.58 & 0.62 & 14.88 & 7.91 & 0.12 & 1.47 & 4.61 & 1.67 & 3.36 & 2.66 & 0.19 & 7.26 & 0.0 & 0.93 & 0.94 & 99.21 \\
\hline 8 & $2-5,47-49$ & 53.58 & 0.62 & 14.75 & 9.00 & 0.11 & 1.67 & 4.45 & 1.63 & 3.53 & 2.94 & 0.29 & 5.17 & 0.0 & 2.29 & 0.80 & 100.83 \\
\hline 9 & $3-1,48-50$ & 54.62 & 0.60 & 15.26 & 7.48 & 0.10 & 0.74 & 3.82 & 1.23 & 2.20 & 2.88 & 0.18 & 7.77 & 0.0 & 0.80 & 1.42 & 99.11 \\
\hline 10 & $3-2,40-42$ & 49.55 & 0.51 & 11.59 & 11.31 & 0.40 & 3.54 & 4.18 & 1.24 & 5.54 & 2.88 & 0.24 & 8.02 & 0.0 & 0.33 & 0.93 & 100.26 \\
\hline II & $3-2,115-117$ & 49.47 & 0.43 & 10.43 & 14.66 & 0.08 & 2.67 & 4.44 & 1.37 & 4.39 & 2.47 & 0.15 & 7.17 & 0.0 & 1.14 & 1.05 & 99.93 \\
\hline 12 & $3-3,6-8$ & 50.66 & 0.48 & 11.84 & 9.82 & 0.08 & 2.66 & 6.07 & 0.96 & 4.01 & 2.22 & 0.16 & 9.23 & 0.0 & 0.61 & 1.90 & 100.69 \\
\hline
\end{tabular}

*(See note at Table 1.)

press b; Sugisaki and Honza, in press), respectively. The samples from the Nankai Trough and its vicinity (Sugisaki, 1978) and from the two areas of the Japan Sea, around the Yamato Bank (Sugisaki, 1979) and the Nishitsugaru Basin (Sugasaki, in press a), are typical terrigenous sediments. The average $\mathrm{SiO}_{2}$ content of the Leg 58 samples amounts to almost 64 per cent, which is much higher than that for the south Philippine Sea and east Pacific Ocean. This indicates that the Shikoku Basin sediments are not typically pelagic, but resemble the terrigenous sediments around the Japanese Islands. This shows an effect on the Shikoku Basin sediments of sediment influx from siliceous continental crust. On the other hand, the $\mathrm{SiO}_{2}$ content of the sediments from the Shikoku Basin is a little lower than that for the Nankai Trough. This indicates that the influence of continental crust on the sediments is reduced southward. In fact, the sediments of the Shikoku Basin are mostly derived from the Japanese Islands. This will be discussed later.

Another aspect of the Leg 58 samples is moderately high manganese content, much higher than in samples from the regions around the Japanese Islands, and lower than in samples from the south Philippine Sea and the east Pacific Ocean. This implies a moderate sedimentation rate in the Shikoku Basin compared to other areas. Sugisaki (1978) suggested that sediments characterized by low Mn and high $\mathrm{Si}$ contents in the Nankai Trough and its vicinity gradually change into pelagic sediments with high $\mathrm{Mn}$ and low Si contents. The present data indicate that such change is proceeding in the region of Leg 58.

\section{HYDROTHERMAL ASPECT OF THE BASAL SEDIMENTS}

The temporal variation in content of some oxides at each site is illustrated in Figure 1. Lithologic units were recognized according to color of the sediments, dominant paleontological and mineralogical components, and sedimentary structures. The deeper parts of the cores tend to be more enriched in $\mathrm{Mn}$ and $\mathrm{Fe}$. The considerable increase in $\mathrm{Mn}$ and $\mathrm{Fe}$ in the oldest sediments is striking, especially at Site 442 . The points representing the oldest sediments at Site 442 in Figure 1 are the average values for four samples collected within 3 meters of the basalt basement (Table 12).
The most likely explanation is that volcanic emanations are responsible for enrichment in $\mathrm{Mn}$ and $\mathrm{Fe}$. Hydrothermal emanations originating at mid-ocean ridges have been thought to provide a substantial source of $\mathrm{Mn}$. Sediments enriched in $\mathrm{Mn}$ and $\mathrm{Fe}$ immediately overlying volcanic basements are found to have been deposited on or near an ocean ridge system with high heat flow (Boström and Peterson, 1969); hot fluids rich in $\mathrm{Fe}, \mathrm{Mn}$, and other metals moved upwards through lavas, giving rise to ferromanganoan deposits. Mineralizing emanations enriched in $\mathrm{Mn}$ and in dissolved gases with a high ratio ${ }^{3} \mathrm{He} /{ }^{4} \mathrm{He}$ were recently found in the Galapagos Rift Zone (Klinkhammer et al., 1977; Lupton et al., 1977). Furthermore, iron-rich mudstones called "umber," directly overlying pillow lavas of the Troodes Massif, Cyprus, are interpreted as precipitates connected with late stages of volcanism on a Cretaceous ocean ridge (Robertson and Hudson 1973). Table 12 also shows the chemical composition of red shale which directly overlies pillow basalts in the Shimanto Belt, Cretaceous geosynclinal area, southwest Japan. These sediments are similar in their high contents of $\mathrm{Mn}$ and Fe. The majority of the Shimanto shales are not associated with basalts and are impoverished in $\mathrm{Mn}$ and $\mathrm{Fe}$. This can be considered evidence for a hydrothermal effect on the basal sediments at Site $\mathbf{4 4 2}$.

On the other hand, the basal sediments at Sites 443 and 444 are not remarkable for their concentrations of $\mathrm{Mn}$ and $\mathrm{Fe}$. The sediments immediately on the basalt basement at Site 442 are paleontologically dated at 15 to 17 m.y., whereas those at Sites 443 and 444 are 14 to 15 m.y. old (Klein et al., 1978). This suggests that the hydrothermal activity waned before deposition at the latter sites.

Boström (1973) empirically found a relationship between the composition of basal sediments and spreading rates at plate-generating zones. The relationship is expressed by the following formula (Boström et al., 1976):

$$
(\mathrm{Fe}+\mathrm{Mn}) / \mathrm{Al}=0.59 \exp (0.53 \mathrm{SR})
$$

where SR is the spreading rate in $\mathrm{cm} / \mathrm{yr}$. If this formula is applied to the Shikoku Basin sediments, the spreading rate at Site 442 is $1.7 \mathrm{~cm} / \mathrm{yr}$, and that at Sites 443 and 444 is $0.5 \mathrm{~cm} / \mathrm{yr}$.

According to the estimation by Kobayashi and Nakada (1978) on the basis of magnetic-anomaly data, the 
TABLE 3

Chemical Composition (\%) of Argillaceous Sediments of Hole 443

\begin{tabular}{|c|c|c|c|c|c|c|c|c|c|c|c|c|c|c|c|c|c|}
\hline No. & $\begin{array}{l}\text { Sample } \\
\text { (interval in } \mathrm{cm} \text { ) }\end{array}$ & $\mathrm{SiO}_{2}$ & $\mathrm{TiO}_{2}$ & $\mathrm{Al}_{2} \mathrm{O}_{3}$ & $\mathrm{Fe}_{2} \mathrm{O}_{3}$ & $\mathrm{FeO}$ & $\mathrm{MnO}$ & $\mathrm{MgO}$ & $\mathrm{CaO}$ & $\mathrm{Na}_{2} \mathrm{O}$ & $\mathrm{K}_{2} \mathrm{O}$ & $\mathrm{P}_{2} \mathrm{O}_{5}$ & $\mathrm{H}_{2} \mathrm{O}$ & $\mathrm{CaCO}_{3}$ & $\begin{array}{l}\text { Resid- } \\
\text { ual* }\end{array}$ & Salt & Total \\
\hline 1 & $443-1-1,125-127$ & 52.06 & 0.61 & 14.88 & 3.58 & 2.03 & 0.53 & 2.94 & 0.61 & 2.05 & 3.04 & 0.12 & 4.07 & 9.21 & 2.86 & 2.00 & 100.59 \\
\hline 2 & $1-4,108-110$ & 57.77 & 0.65 & 15.92 & 3.33 & 2.28 & 0.29 & 2.38 & 0.39 & 1.60 & 3.05 & 0.15 & 4.30 & 1.94 & 2.45 & 2.57 & 99.07 \\
\hline 3 & $2-1,71-73$ & 58.11 & 0.68 & 15.48 & 3.79 & 2.14 & 0.44 & 2.71 & 0.63 & 1.82 & 3.08 & 0.17 & 4.10 & 1.47 & 2.83 & 2.67 & 100.11 \\
\hline 4 & $2-3,45-47$ & 59.11 & 0.72 & 14.52 & 3.58 & 2.42 & 0.16 & 3.08 & 0.16 & 1.61 & 3.42 & 0.12 & 5.35 & 1.88 & 1.48 & 1.46 & 99.06 \\
\hline 5 & $3-1,16-18$ & 60.37 & 0.73 & 14.92 & 3.30 & 2.34 & 0.20 & 3.06 & 1.05 & 1.32 & 3.47 & 0.13 & 2.62 & 0.0 & 4.10 & 1.46 & 99.06 \\
\hline 6 & $3-2,77-79$ & 61.89 & 0.73 & 15.08 & 3.02 & 2.42 & 0.16 & 2.96 & 0.13 & 1.04 & 3.45 & 0.11 & 4.38 & 1.90 & 1.91 & 1.63 & 100.82 \\
\hline 7 & $3-5,84-86$ & 57.30 & 0.67 & 13.87 & 3.35 & 2.31 & 0.56 & 3.19 & 0.33 & 1.77 & 3.37 & 0.12 & 5.19 & 5.51 & 0.22 & 2.19 & 99.96 \\
\hline 8 & $4-1,111-113$ & 58.72 & 0.66 & 13.93 & 3.42 & 2.15 & 0.43 & 2.66 & 0.02 & 1.42 & 3.31 & 0.12 & 2.71 & 6.08 & 2.52 & 2.27 & 100.42 \\
\hline 9 & $4-2,109-111$ & 52.88 & 0.62 & 14.78 & 3.74 & 2.05 & 0.46 & 2.48 & 1.42 & 1.26 & 3.04 & 0.11 & 5.20 & 5.74 & 2.91 & 2.34 & 99.03 \\
\hline 10 & $4-3,39-41$ & 61.80 & 0.72 & 16.76 & 3.06 & 2.41 & 0.17 & 2.39 & 0.15 & 1.11 & 3.22 & 0.13 & 4.72 & 1.72 & 0.52 & 2.13 & 101.00 \\
\hline 11 & $4-3,45-47$ & 56.03 & 0.66 & 15.60 & 3.09 & 2.14 & 0.34 & 2.36 & 0.18 & 1.53 & 3.01 & 0.15 & 3.56 & 6.88 & 1.85 & 2.18 & 99.57 \\
\hline 12 & $5-1,61-63$ & 56.93 & 0.71 & 16.29 & 3.48 & 2.30 & 0.25 & 2.64 & 0.41 & 1.77 & 3.28 & 0.13 & 6.37 & 2.95 & 0.77 & 1.45 & 99.72 \\
\hline 13 & $5-2,36-38$ & 55.80 & 0.69 & 15.84 & 3.69 & 2.25 & 0.41 & 2.79 & 0.14 & 1.63 & 3.18 & 0.11 & 6.47 & 4.88 & 0.25 & 1.35 & 99.48 \\
\hline 14 & $5-3,99-101$ & 53.27 & 0.64 & 14.80 & 3.66 & 2.12 & 0.35 & 2.69 & 0.07 & 1.78 & 3.00 & 0.12 & 5.11 & 7.71 & 2. & 1.67 & 99.12 \\
\hline 15 & $5-4,31-33$ & 57.43 & 0.58 & 14.89 & 2.91 & 2.20 & 0.37 & 2.01 & 2.67 & 1.99 & 2.93 & 0.12 & 6.59 & 2.73 & 0.28 & 1.79 & 99.48 \\
\hline 16 & $6-2,44-46$ & 58.02 & 0.60 & 15.55 & 3.02 & 2.04 & 0.36 & 2.06 & 0.26 & 2.12 & 2.90 & 0.13 & 4.33 & 6.79 & 0.65 & 1.85 & 100.69 \\
\hline 17 & $6-3,44-46$ & 42.89 & 0.50 & 11.64 & 2.99 & 1.57 & 0.56 & & 5.48 & 2.01 & 2.49 & 0.13 & 4.65 & 14.67 & & 1.36 & 98.39 \\
\hline 18 & $6-4,44-46$ & 62.94 & 0.63 & 16.01 & 2.63 & 2.05 & 0.11 & 1.94 & 1.30 & 1.84 & 3.14 & 0.10 & 6.30 & 0.0 & 0.91 & 1.08 & 100.99 \\
\hline 19 & $6-5,44-46$ & 57.43 & 0.66 & 15.78 & 3.45 & 2.12 & 0.20 & 2.28 & 0.60 & 1.38 & 3.05 & 0.13 & 6.27 & 3.85 & 0.28 & 1.32 & 99.01 \\
\hline 20 & $8-4,106-108$ & 53.95 & 0.65 & 15.08 & 3.61 & 2.28 & 0.44 & 2.33 & 0.03 & 1.10 & 3.13 & 0.16 & 5.45 & 8.01 & 1.29 & 1.95 & 99.45 \\
\hline 21 & $8-5,58-60$ & 57.35 & 0.71 & 16.27 & 3.51 & 2.28 & 0.33 & 2.85 & 0.40 & 1.38 & 3.32 & 0.12 & 5.80 & 3.05 & 0.78 & 1.46 & 99.60 \\
\hline 22 & $9-3,28-30$ & 53.36 & 0.67 & 15.33 & & 1.99 & 0.25 & 2.47 & 0.27 & 1.05 & & & 6.26 & 8.01 & & & 99.28 \\
\hline 23 & $9-5,26-28$ & 58.56 & 0.71 & 16.71 & 3.57 & 2.22 & 0.19 & 2.65 & 0.15 & 1.24 & & 0.11 & 5.66 & 3.52 & 0. & & 99.96 \\
\hline 24 & $10-1,69-71$ & 58.11 & 0.67 & 16.84 & 3.94 & 1.97 & 0.28 & 2.44 & 0.13 & 1.53 & 3.05 & 0.12 & 5.84 & 2.20 & 0 . & 1. & 99.20 \\
\hline 25 & $10-1,138-140$ & 57.91 & 0.65 & 15.90 & 3.90 & 2.01 & 0.27 & 2.50 & 0.34 & 1.36 & 3.05 & 0.12 & 6.51 & 2.78 & 0.01 & 1.59 & 98.89 \\
\hline 26 & $10-2,39-41$ & 60.16 & 0.62 & 15.02 & 3.82 & 1.90 & 0.27 & 2.50 & 1.64 & 1.53 & 2.93 & 0.11 & 6.20 & 0.0 & 1.02 & 1.34 & 99.05 \\
\hline 27 & $10-2,138-140$ & 58.00 & 0.65 & 15.85 & 3.95 & & 0.29 & & 0.60 & 1.83 & & & 6.70 & 0 & & & 100.36 \\
\hline 28 & $10-3,43-45$ & 57.43 & 0.64 & 15.87 & 4.29 & 2.07 & 0.27 & 7 & 1.38 & 1.36 & 3.07 & 0.11 & 7.92 & 0.0 & 0.47 & 1.58 & 99.03 \\
\hline 29 & $10-4,51-53$ & 58.45 & 0.66 & 15.97 & 4.32 & 1.93 & 0.24 & 2.31 & 0.69 & 1.89 & 3.11 & 0.13 & 6.21 & 2.06 & 0.32 & 1. & 99.95 \\
\hline 30 & $10-4,135-137$ & 56.95 & 0.66 & 16.07 & 3.86 & 2.12 & 0.26 & 2.68 & 0.17 & 1.49 & 3.11 & 0.12 & 6.54 & 3.08 & 0.42 & 1.53 & 99.07 \\
\hline 31 & $10-5,48-50$ & 56.00 & 0.66 & 16.00 & 4.20 & 1.97 & 0.30 & 2.69 & 0.40 & 1.66 & 3.18 & 0.11 & 5.96 & 4.17 & 0.09 & 1.88 & 99.27 \\
\hline 32 & $10-5,132-134$ & 54.05 & 0.66 & 14.67 & 3.92 & 1. & 0.42 & 2.63 & 0.18 & 1.69 & & & 4.91 & & & & 99.02 \\
\hline 33 & $10-6,108-110$ & 59.29 & 0.54 & 14.68 & 4.29 & 1.8 & 0.12 & 2.31 & 0.07 & 1.35 & 3. & 0. & 5.95 & 3. & & 1.14 & 99.07 \\
\hline 34 & $11-2,108-110$ & 52.04 & 0.63 & 14.62 & 3.42 & 2.01 & 0.36 & 2.27 & 0.46 & 1.97 & 3. & 0.13 & 4.86 & 12.73 & 0.35 & 1.39 & 100.34 \\
\hline 35 & $11-3,108-110$ & 53.01 & 0.63 & 14.73 & 3.94 & 2.07 & 0.60 & 2.61 & 0.19 & 2.29 & 2.97 & 0.17 & 4.68 & 9.47 & 1.09 & 2 & 99.97 \\
\hline 36 & $11-4,1$ & 59.03 & 0.69 & .25 & 1 & 2. & 0.17 & 2.64 & 0.21 & 1.55 & 3.22 & 0.11 & 6.41 & 2.01 & 1.72 & 1.3 & 101.10 \\
\hline 37 & $11-5,108-110$ & 58.79 & 0.64 & 16.00 & 3.53 & 2.05 & 0.38 & 2 & 0 . & 1.84 & 7 & 0. & 6.72 & 1. & 0.8 & 1. & 99.97 \\
\hline 38 & $11-6,70-72$ & 59.25 & 0.71 & 16.32 & 3.55 & 2.24 & 0.14 & 2.52 & 1.30 & 1.61 & 3.1 & 0.11 & 5.88 & 0 . & 1. & 1. & 99.13 \\
\hline 39 & $14-4,120-122$ & 57.48 & 0.71 & 16.85 & 4.16 & 2.25 & 0.30 & 2.58 & 1.08 & 1.75 & 3.27 & 0.11 & 6.78 & 0.0 & 0.39 & & 99.11 \\
\hline 40 & $14-5,120-122$ & 58.86 & 0.65 & 16.23 & 4.05 & 1.99 & 0.08 & 2.20 & 1.22 & 1.38 & 3.29 & 0.097 & 5.53 & 0.0 & 2.38 & 1.21 & 99.17 \\
\hline 41 & $14-6,106-108$ & 59.73 & 0.70 & 16.53 & 3.33 & & 0.16 & 12 & 0.55 & 1.57 & 3.12 & 0.12 & 5.78 & 2.49 & 0.45 & 1.21 & 100.14 \\
\hline 42 & $15-4,48-50$ & 59.23 & 0.76 & 17.72 & 19 & 2. & 0.14 & 3 & 0.27 & 1.57 & & & 5.96 & 2.03 & 0. & 1. & 100.87 \\
\hline 43 & $15-5,63-65$ & 56.30 & 0.70 & 16.63 & 4.47 & 2.19 & 0.60 & 2.91 & 0.37 & 1.95 & 3. & 0 . & 5.84 & 1.29 & 0 . & 1. & 98.58 \\
\hline 44 & $15-6,63-65$ & 57.22 & 0.72 & 17.10 & 4.11 & 2.15 & 0.30 & 2.80 & 0.24 & 1.52 & 3.33 & 0.12 & 5.92 & 1.46 & 0.67 & & 99.03 \\
\hline 45 & $15-7,2$ & 57.47 & 0.71 & 16.89 & 4.06 & 2.16 & 0.28 & 2.66 & 0.30 & 1.16 & 3.18 & & 6.69 & & 0.14 & & 99.06 \\
\hline 46 & t & .81 & 0.70 & 15.95 & 3.77 & 2.11 & 0.45 & 2.46 & 0.16 & 1.35 & 3.15 & 0.11 & 6.03 & 8.08 & 0.61 & 1.50 & 99.24 \\
\hline 47 & $18-1,98-100$ & .12 & 68 & 18 & 4 & 2. & 0 . & 1 & 1. & 1. & & & 8 & & & & 97 \\
\hline 48 & $18-2,98-100$ & 59.47 & 0.61 & 16.30 & 3.47 & 1.89 & 0.14 & 2.01 & 0.18 & 1.31 & 3. & & 6.88 & 2.47 & 0.10 & & 99.56 \\
\hline 49 & $18-3,98-100$ & 64.22 & 0.42 & 13.95 & 2.07 & 1.47 & 0.17 & 1.14 & 1.20 & 1.88 & 2.73 & 0.083 & 4.29 & 3.17 & & 0.97 & 99.24 \\
\hline 50 & $21-2,13-15$ & 56.04 & 0.72 & 17.19 & 4.71 & 1.75 & 0.55 & 2.97 & 0.24 & 1.72 & 3.21 & & 6.49 & 2.00 & & 1.42 & 99.48 \\
\hline 51 & $23-1,110-112$ & 56.61 & 0.70 & 16.70 & 5.15 & 1.48 & 036 & 2.80 & 0.25 & 2.99 & 3.21 & 0.12 & 6.77 & 2.06 & 0.30 & 1.02 & 100.52 \\
\hline 52 & $23-2,9$ & 37 & 63 & 16.17 & 4. & & & 2.48 & & & & & 6.35 & & & & 100.08 \\
\hline 53 & $23-3,40-42$ & 56.72 & 0.48 & 14.89 & 3.50 & 1.24 & 0.31 & 1.66 & 0.29 & 1.53 & 3. & 98 & 4.86 & 8.00 & & & 98.92 \\
\hline 54 & $23-4,50-52$ & 59.96 & 0.54 & 15.83 & 3.95 & 1.38 & 0.33 & 2.12 & 0.19 & 1.58 & & 0.095 & 7.14 & 1.88 & & & 99.60 \\
\hline 55 & 17077 & 55.66 & 0.69 & 16.58 & 5.19 & 1.66 & 0.51 & 3.14 & 0.87 & 1.74 & 2.8 & & 6.72 & & 0.16 & 1.18 & 100.33 \\
\hline 56 & $-3,42-44$ & 54.91 & 0.70 & 16.68 & 186 & 1.67 & 0.28 & 2.89 & 0.49 & 1.21 & 303 & 0 & 5.95 & 3.23 & 1.37 & 1.63 & 99.05 \\
\hline 57 & $24-3,90$ & & 0.72 & 16.42 & & & & & & & & & 8 & & & & 99.08 \\
\hline 58 & $24-4,95-97$ & 55.26 & 0.69 & 16.58 & 5.1 & 1.77 & 0.3 & 2.99 & 0.43 & 1.89 & 2.8 & & 6.69 & & & & 99.38 \\
\hline 59 & $24-5,71-73$ & 50.63 & 0.66 & 14.66 & 5.41 & 2.40 & 0.2 & 2.84 & 1.5 & 1.17 & 2.11 & & 3.34 & 10.23 & & 1.96 & 99.02 \\
\hline 60 & $24-6,74-76$ & 46.67 & 0.61 & 13.98 & 4.75 & 1.39 & 0.61 & 2.46 & 0.30 & 1.27 & 2.53 & & 5.24 & & & & 100.85 \\
\hline 61 & $25-2,7$ & 50.13 & 0.85 & 14.29 & 5.1 & 4.40 & 0.22 & 3.02 & 4.51 & 0.8 & 1.0 & & 2.46 & 10. & 0.46 & 1.10 & 99.00 \\
\hline 62 & $25-2,123-125$ & & 0.57 & 13 & 4.2 & 1. & 0.5 & 2.38 & & & & & 2.71 & 20.87 & & & 101.47 \\
\hline 63 & $25-3,86-88$ & 56.56 & 0.69 & 16.34 & 5.01 & 2.09 & 0.32 & 2.73 & 2.30 & 1.30 & 2. & 0 . & 6.31 & 2.85 & 0.36 & & 101.07 \\
\hline 64 & $26-1,82-84$ & 57.14 & 0.67 & 16.27 & 4.80 & 2.21 & 0.38 & 2.53 & 2.58 & 1.67 & 2.52 & & 5.69 & 0.0 & & 1.20 & 99.01 \\
\hline 65 & $26-2,142-144$ & 50.11 & 0.64 & 14.19 & 4.80 & 2.07 & 0.48 & 2.53 & 0.13 & 1.78 & 2.32 & 0.12 & 3.22 & 14.46 & 1.69 & 1.09 & 99.64 \\
\hline
\end{tabular}


TABLE 3 - Continued

\begin{tabular}{|c|c|c|c|c|c|c|c|c|c|c|c|c|c|c|c|c|c|}
\hline No. & $\begin{array}{c}\text { Sample } \\
\text { (interval in } \mathrm{cm} \text { ) }\end{array}$ & $\mathrm{SiO}_{2}$ & $\mathrm{TiO}_{2}$ & $\mathrm{Al}_{2} \mathrm{O}_{3}$ & $\mathrm{Fe}_{2} \mathrm{O}_{3}$ & $\mathrm{FeO}$ & $\mathrm{MnO}$ & $\mathrm{MgO}$ & $\mathrm{CaO}$ & $\mathrm{Na}_{2} \mathrm{O}$ & $\mathrm{K}_{2} \mathrm{O}$ & $\mathrm{P}_{2} \mathrm{O}_{5}$ & $\mathrm{H}_{2} \mathrm{O}$ & $\mathrm{CaCO}_{3}$ & $\begin{array}{l}\text { Resid- } \\
\text { ual* }\end{array}$ & Salt & Total \\
\hline 66 & $443-27-1,80-82$ & 60.32 & 0.58 & 14.88 & 3.80 & 1.94 & 0.28 & 2.10 & 0.62 & 2.07 & 2.34 & 0.12 & 6.52 & 2.26 & 0.17 & 1.31 & 99.32 \\
\hline 67 & $27-2,22-24$ & 55.37 & 0.65 & 15.65 & 5.23 & 1.49 & 0.83 & 2.90 & 0.53 & 2.28 & 2.85 & 0.16 & 5.94 & 4.01 & 1.07 & 1.37 & 100.33 \\
\hline 68 & $27-3,11-13$ & 57.64 & 0.59 & 15.89 & 1.56 & 1.41 & 1.33 & 2.98 & 0.12 & 3.64 & 2.94 & 0.21 & 3.95 & 4.25 & 2.32 & 1.12 & 99.94 \\
\hline 69 & $27-4,55-57$ & 55.62 & 0.68 & 16.37 & 4.95 & 1.48 & 0.25 & 2.43 & 1.19 & 1.10 & 3.00 & 0.15 & 7.62 & 0.0 & 2.16 & 1.10 & 98.09 \\
\hline 70 & $28-1,108-110$ & 60.30 & 0.69 & 17 & 5.13 & 1.44 & 0.10 & 2.20 & 0.96 & 1.04 & 3.08 & 0.13 & 6.35 & 0.0 & 1.45 & 0.85 & 100.93 \\
\hline 71 & $28-2,16-18$ & 58.35 & 0.67 & 16.94 & 5.12 & 1.38 & 0.29 & 2.52 & 1.03 & 1.52 & 3.12 & 0.14 & 5.57 & 0.0 & 1.58 & 1.09 & 99.32 \\
\hline 72 & $29-1,87-89$ & 57.21 & 0.65 & & 4.75 & 1.46 & 0.43 & 2.46 & 0.19 & 1.82 & 2.83 & & 6.79 & & 0.69 & & \\
\hline 73 & $29-2,61-63$ & 56.96 & 0.67 & 17.26 & 5.10 & 1.44 & 0.37 & 2.64 & 1.11 & 1.08 & 3.05 & 0.12 & 6.27 & 0.0 & 1.17 & 1.84 & 99.08 \\
\hline 74 & $29-3,85-87$ & 56.64 & 0.67 & 16.89 & 4.5 & 1.60 & 0.39 & 2.58 & 0.49 & 4.77 & 3.04 & 0.14 & 5.61 & 1.37 & 0.95 & 1.21 & 100.92 \\
\hline 75 & $29-4,56-58$ & 58.03 & 0.61 & 17.08 & 4.98 & 1.71 & 0.39 & 2.65 & 1.52 & 1.90 & 2.99 & 0.15 & 5.96 & 0.0 & 1.86 & 1.19 & 101.03 \\
\hline 76 & $29-5,46-48$ & 57.00 & 0.67 & 16.74 & 5.39 & 1.53 & 0.54 & 2.74 & 0.08 & 2.08 & 2.88 & 0.14 & 6.28 & 2.99 & 0.00 & 1.23 & 100.29 \\
\hline 77 & $30-2,132-134$ & 57.10 & 0.63 & 15. & 4. & 1.62 & & 3.06 & & & & & & 3.27 & & & \\
\hline 78 & $31-1,119-121$ & 57.64 & 0.66 & 15. & 5.0 & 1.86 & 0.13 & 2.44 & 2.46 & 1.65 & 2.43 & 0.13 & 6.93 & 0.0 & & & \\
\hline 79 & $31-2,62-64$ & 57.56 & 0.67 & 16.12 & 4.96 & 2.10 & 0.48 & 2.86 & 1.91 & 2.71 & 2.30 & 0.14 & 5.16 & 2.57 & 0.55 & 99 & 101.08 \\
\hline 80 & 3 & 56.84 & 0.66 & 15.35 & 4.84 & 2.34 & 0.45 & 2.90 & 1.44 & 1.97 & 2.42 & 0.14 & 5.19 & 2.40 & 1.19 & 1.23 & 36 \\
\hline 81 & $31-4,44-46$ & 56.67 & 0.65 & 15.42 & 4.91 & 1.95 & 0.54 & 2.82 & 1.72 & 2.29 & 2.51 & 0.14 & 6.06 & 1.86 & 0.33 & 1.20 & 99.07 \\
\hline 82 & $-5,30-32$ & .49 & 0.6 & 15.1 & 4.9 & 2.02 & & 2.54 & 1.03 & 2. & 2.51 & 0.12 & & 2.61 & 0 & & \\
\hline 83 & $32-1,129-131$ & 52.52 & 0.65 & 14.93 & 4.70 & 2.28 & 0.51 & 2.43 & 2.24 & 2.34 & 2.04 & 0.12 & 5.22 & 7.41 & & 5 & oc \\
\hline 84 & $-1,38-40$ & 52.39 & 0.76 & 14.54 & 4.71 & 2.26 & 0. & 2.31 & 1.75 & 2.15 & 2. & 0. & 5.08 & 9.35 & 0.31 & 1.02 & 99 \\
\hline 85 & $33-2,38-40$ & 62.57 & 0.40 & 13.54 & 2.85 & 1.63 & 0.22 & 1.35 & 2.38 & 2.50 & 3.36 & 0.085 & 5.47 & 0.0 & 1.68 & 1.03 & 99.06 \\
\hline 86 & $-3,3$ & 51.98 & 0.64 & 14.10 & 4.70 & 1.92 & 0.35 & 2.12 & 1.83 & 2.08 & 2.22 & 0.13 & 4.66 & 11.02 & 0.28 & 1.05 & 99.08 \\
\hline 87 & & & & & & & & & & & & & & & & & \\
\hline 88 & $-3,11-13$ & 3.27 & 0.5 & 13 & 48 & 1. & 0. & 2 & 1. & 2. & 2 & 0 & & 6 & 0 & 1.20 & 99 \\
\hline 89 & $4,11-13$ & 45.42 & 0.48 & & 3. & 1.2 & & & & & & & & 01 & & 1.3 & 99 \\
\hline 90 & $11-13$ & 55.45 & 0.64 & 14.75 & 4.1 & 2.05 & 0.16 & 2.31 & 1.81 & 1.86 & 2.03 & 0. & 4.35 & 7.41 & 0.71 & 1.24 & 99.01 \\
\hline 91 & & 45.07 & 0.41 & 10.9 & 3.5 & 1.53 & 0.3 & 1.53 & 0.47 & 1.99 & 2.22 & 0. & 2.73 & 27.23 & 0.76 & 1.07 & 99.93 \\
\hline 92 & & 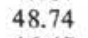 & & 1 & & & & & & & & & & & & & \\
\hline 93 & $36-3$ & 5.47 & 0.6 & 12 & 4.7 & 1. & & & & & & & & & & & 98 \\
\hline 94 & -105 & 4 & 0.5 & 11. & 4.5 & 1. & & & & & & & & & & & $a c$ \\
\hline 95 & & 40 & 0. & 11 & 4. & 1.3 & 1.5 & 2.62 & 7.56 & 2.64 & 2.0 & 0. & 4.30 & 5 & 4 & 90 & 10 \\
\hline 96 & 16 & 55.65 & $0.6-1-1$ & 16 & 5.4 & 1.7 & 0. & 4. & 2.67 & 1.4 & 2.73 & & 6.51 & & 9 & .80 & \\
\hline 97 & & & & & & & & & & & & & & & & 9 & \\
\hline 98 & , $76-78$ & 54.01 & & 15. & & 2.02 & & & & & & & & & & & \\
\hline 99 & -131 & & & & 4.5 & 1. & & & & & & & & & & 7 & 100.82 \\
\hline 100 & 3 & 39.78 & 0.4 & 10.22 & 3.74 & 1.39 & 3.01 & 2.43 & 4.41 & 3.8 & 1.88 & 1.1 & 4.07 & 21.00 & 3.59 & 1.41 & 102.37 \\
\hline 101 & , & 58 & 0 & 16.12 & 6.26 & 1.64 & 0.17 & 2.85 & 2.23 & 1.57 & 2.73 & 0. & 7 . & 0.0 & 0.58 & 1: & 99 \\
\hline & 4 & & & & & & & & & & & & & & & & \\
\hline 03 & 166 & 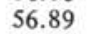 & & & & & & & & & & & & & & & 10 \\
\hline 10 & $13-15$ & 5 & & 15. & 5. & & & & 0. & & & & & & & & \\
\hline 105 & $43-1,38-40$ & 59.01 & 0.6 & 15. & 4.8 & 1.6 & 0. & 2.64 & 1.86 & 1. & 2.91 & 0.1 & 6.73 & 0. & 0.68 & 1.21 & 99.05 \\
\hline 106 & $78-80$ & 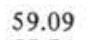 & 061 & 14.5 & 4.3 & 2.1 & 2. & 2. & 2. & 1 . & 287 & 0.1 & 589 & 0 & 0.73 & 1.57 & 100.84 \\
\hline & & & & & & & & & & & & & & & & 25 & \\
\hline 1 & & & & & & & & & & & & & & & & & 99.52 \\
\hline 10 & $-1,44-46$ & & 0. & 11 & & 1.6 & & & 0.62 & & & & & 20.56 & & & \\
\hline 110 & $44-46$ & 56.59 & 0.66 & & 6.21 & 1.81 & 0.75 & 3.17 & 1.08 & 3.00 & 2.42 & 0.12 & 5.78 & 3.08 & 0.12 & 1.29 & 100.80 \\
\hline 111 & $126-3$ & 57.94 & 0.62 & 14 & 6.0 & 1.74 & 0 . & 2. & 2.15 & 1.52 & 2.57 & 0.10 & 6.42 & 0 & 0.84 & 1.21 & 99.08 \\
\hline 112 & & & & & & 1. & & & 0.07 & & & & & & & & \\
\hline 113 & & 55.1 & 0. & 13 & 9. & 0.5 & 0. & & 0. & 1. & 2. & 0. & 6. & & 0.52 & & \\
\hline 114 & $49-2,114-116$ & 35.90 & 0.45 & 9.67 & 6.99 & 0.30 & 0.73 & 3.27 & 4.52 & 0.59 & 1.85 & 0.14 & 6.15 & 28.11 & 1.78 & 0.55 & 101.00 \\
\hline
\end{tabular}

*(See note at Table 1.)

spreading rate of the Shikoku Basin was 4 to $6 \mathrm{~cm} / \mathrm{yr}$ between 26 and $22 \mathrm{Ma}$, and it subsequently decreased to about half that rate $(2-3 \mathrm{~cm} / \mathrm{yr})$. Rates after $18 \mathrm{Ma}$ seem to vary with time and place. Watts and Weissel (1975) estimated the rate between 22 and $19 \mathrm{Ma}$ to be 2.2 $\mathrm{cm} / \mathrm{yr}$. The rate estimated from chemical parameters at Site 442 is apparently not inconsistent with the geophysical estimate for the last period of spreading. Spreading slowed during deposition of the basal sediments at Sites 443 and 444 (15-14 Ma), if the chemical parameters provide a valid indication.

\section{POSSIBLE SOURCES OF THE SEDIMENTS IN THE SHIKOKU BASIN}

Seismic profiles obtained by Murauchi and Asanuma (1977), Karig (1975), and others showed that there are three coalescing clastic wedges in the basin. A wedge on the north side of the basin was penetrated at DSDP Site 297; a second, on the west side of the basin, was penetrated at Site 442; and the third, on the east side, was penetrated at Sites 443 and 444 . The direction of thickening of these wedges suggests that the north 
TABLE 4

Chemical Composition (\%) of Argillaceous Sediments of Hole 444

\begin{tabular}{|c|c|c|c|c|c|c|c|c|c|c|c|c|c|c|c|c|c|}
\hline No. & $\begin{array}{c}\text { Sample } \\
\text { (interval in } \mathrm{cm} \text { ) }\end{array}$ & $\mathrm{SiO}_{2}$ & $\mathrm{TiO}_{2}$ & $\mathrm{Al}_{2} \mathrm{O}_{3}$ & $\mathrm{Fe}_{2} \mathrm{O}_{3}$ & $\mathrm{FeO}$ & $\mathrm{MnO}$ & $\mathrm{MgO}$ & $\mathrm{CaO}$ & $\mathrm{Na}_{2} \mathrm{O}$ & $\mathrm{K}_{2} \mathrm{O}$ & $\mathrm{P}_{2} \mathrm{O}_{5}$ & $\mathrm{H}_{2} \mathrm{O}$ & $\mathrm{CaCO}_{3}$ & Residual* & Salt & Total \\
\hline 1 & $444-3-1,12-14$ & 59.40 & 0.68 & 16.10 & 3.54 & 2.27 & 0.26 & 2.58 & 0.57 & 1.94 & 2.95 & 0.14 & 5.05 & 1.81 & 0.19 & 1.52 & 99.00 \\
\hline 2 & $3-2,22-24$ & 56.10 & 0.68 & 15.83 & 4.05 & 2.09 & 0.77 & 3.54 & 0.35 & 2.10 & 3.19 & 0.13 & 5.10 & 3.53 & 1.72 & 1.59 & 100.76 \\
\hline 3 & $3-3,20-22$ & 58.70 & 0.73 & 16.13 & 3.91 & 2.22 & 0.17 & 2.59 & 0.66 & 1.30 & 3.12 & 0.14 & 5.83 & 1.26 & 0.52 & 1.78 & 99.07 \\
\hline 4 & $4-1,116-118$ & 57.58 & 0.72 & 16.24 & 3.59 & 1.99 & 0.21 & 2.78 & 0.11 & 1.59 & 3.29 & 0.12 & 5.46 & 2.08 & 1.27 & 1.79 & 98.82 \\
\hline 5 & $4-2,116-118$ & 59.28 & 0.70 & 15.89 & 4.24 & 2.06 & 0.13 & 2.59 & 0.58 & 1.57 & 3.05 & 0.14 & 5.66 & 1.59 & 0.17 & 1.66 & 99.30 \\
\hline 6 & $4-3,20-22$ & 57.93 & 0.73 & 16.37 & 4.63 & 1.97 & 0.17 & 2.52 & 0.21 & 1.38 & 3.19 & 0.12 & 6.25 & 1.68 & 0.94 & 1.37 & 99.46 \\
\hline 7 & $4-3,114-116$ & 56.04 & 0.67 & 15.34 & 4.61 & 1.48 & 0.60 & 2.11 & 0.68 & 2.43 & 2.90 & 0.15 & 5.05 & 3.99 & 1.49 & 1.50 & 99.03 \\
\hline 8 & $5-1,76-78$ & 58.90 & 0.72 & 15.70 & 4.15 & 2.54 & 0.21 & 2.33 & 2.56 & 1.93 & 2.45 & 0.13 & 5.12 & 0.0 & 0.83 & 1.36 & 98.92 \\
\hline 9 & $5-2,92-94$ & 56.07 & 0.69 & 16.12 & 4.54 & 1.74 & 0.22 & 2.45 & 0.61 & 1.37 & 3.14 & 0.13 & 5.99 & 6.28 & 0.07 & 1.51 & 100.92 \\
\hline 10 & $5-3,60-62$ & 56.52 & 0.74 & 16.82 & 4.14 & 2.20 & 0.48 & 3.05 & 0.31 & 1.92 & 3.11 & 0.15 & 5.28 & 2.15 & 1.38 & 1.59 & 99.85 \\
\hline 11 & $5-4,68-70$ & 56.96 & 0.70 & 16.42 & 3.64 & 2.83 & 0.20 & 2.64 & 1.01 & 1.74 & 3.08 & 0.16 & 5.99 & 2.01 & 0.03 & 1.59 & 99.00 \\
\hline 12 & $7-1,50-52$ & 57.86 & 0.72 & 16.55 & 4.81 & 1.72 & 0.25 & 2.50 & 1.70 & 1.58 & 2.89 & 0.15 & 6.42 & 0.0 & 0.39 & 1.53 & 99.08 \\
\hline 13 & $7-2,77-79$ & 58.09 & 0.72 & 16.62 & 4.76 & 1.85 & 0.21 & 2.68 & 1.60 & 1.95 & 2.97 & 0.14 & 6.87 & 0.0 & 0.41 & 1.50 & 100.37 \\
\hline 14 & $7-3,43-45$ & 60.07 & 0.65 & 15.22 & 3.48 & 1.81 & 0.33 & 2.08 & 2.12 & 2.86 & 2.74 & 0.13 & 6.19 & 0.0 & 0.26 & 1.10 & 99.03 \\
\hline 15 & $7-4,12-14$ & 62.10 & 0.64 & 16.07 & 3.74 & 1.63 & 0.16 & 2.19 & 1.83 & 1.86 & 3.03 & 0.12 & 6.13 & 0.0 & 0.36 & 1.25 & 101.10 \\
\hline 16 & $7-6,28-30$ & 57.55 & 0.72 & 16.74 & 4.59 & 2.11 & 0.13 & 2.38 & 2.00 & 1.17 & 2.85 & 0.16 & 5.44 & 0.0 & 1.39 & 1.69 & 98.92 \\
\hline 17 & $9-1,110-112$ & 60.71 & 0.64 & 14.82 & 3.35 & 2.67 & 0.15 & 2.18 & 3.80 & 2.02 & 2.20 & 0.15 & 5.20 & 0.0 & 0.33 & 1.09 & 99.31 \\
\hline 18 & $10-1,52-54$ & 58.60 & 0.64 & 16.08 & 4.38 & 1.71 & 0.19 & 2.62 & 1.75 & 1.95 & 2.73 & 0.14 & 7.31 & 0.0 & 0.51 & 1.45 & 100.05 \\
\hline 19 & $10-2,52-54$ & 56.22 & 0.68 & 16.34 & 4.75 & 1.70 & 0.32 & 2.91 & 1.73 & 1.67 & 3.00 & 0.15 & 6.61 & 0.0 & 1.23 & 1.78 & 99.09 \\
\hline
\end{tabular}

*(See note at Table 1.)

wedge was probably derived from the Japanese Islands, whereas the west and east wedges were derived from the Kyushu-Palau Ridge and the Iwo Jima Ridge, respectively. Geochemical data obtained in the present study can be used to evaluate these possibilities.

The relationships among $\mathrm{SiO}_{2}, \mathrm{TiO}_{2}$ and $\mathrm{Al}_{2} \mathrm{O}_{3}$ (Figure 2) are helpful in the examination of the sediment source, because these components tend to remain in the resistates and hydrolysates during sedimentation (e.g., Rankama and Sahama, 1950), and their relative abundances are preserved during weathering. The points in Figure 2 fall along a line connecting points for averaged Japanese granites and averaged Quaternary volcanics. Points for averaged shales in the Shimanto Belt (Cretaceous geosynclinal area) and the Chichibu terrain (Paleozoic geosynclinal area), the Japanese Islands, also plot near the line. The point for Izu-Mariana volcanics plots off the line; this strongly suggests that the Shikoku Basin sediments were mostly derived from the Japanese Islands, and not from the Izu-Mariana Islands. Sediments from the east Pacific Ocean and the Philippine Sea plot in an area of lower silica. The $\mathrm{SiO}_{2}$ content of Nankai Trough sediments, which were also derived from the Japanese Islands (Sugisaki, 1978), is a little higher than that of the Leg 58 samples (Table 11). Silica in sediments off the coast of southwest Japan tends to decrease southward, probably because of the influence of oceanic and basic materials derived from islands such as the Izu-Mariana islands. Such local contribution to the Shikoku Basin sediments, however, is not as large as expected from seismic evidence. The closely similar plots for the three sites on the ternary diagrams of Figure 2 reinforce the inference.

The temporal variation in $\mathrm{SiO}_{2}$ content at each site is rather small and does not show any trend (Figure 1), indicating continuous influx of terrigenous sediments from the north. Karig (1975) presumed that the Pliocene development of the Nankai Trough as a sediment trap reduced or completely eliminated the supply of the sedi- ments from the north. However, the geochemical data of the sediments show that the Nankai Trough cannot have been a barrier to sediment influx from the north.

\section{CHEMICAL ALTERATION OF THE SEDIMENTS}

The sediments must have undergone some alteration during diagenesis. To what extent was the original composition of the sediments changed? The sediments in the Japan Trench were observed to be considerably contaminated by biogenic silica (Sugisaki, in press c) and they are not suitable for evaluating chemical alteration. On the other hand, Shikoku Basin samples with a small amount of silica contamination will serve the present purpose.

Figure 3 exemplifies the relationships among $\mathrm{TiO}_{2}$, total $\mathrm{Fe}$ as $\mathrm{Fe}_{2} \mathrm{O}_{3}$, and $\mathrm{MgO}$. The averaged variations of these elements in granites and volcanics in the Japanese Islands were plotted in the figure together with some averaged sediments from various regions. General aspects emerge from the plot: (1) Most sediments of the Shikoku Basin, which plot between the averaged volcanics and granites, originated in the Japanese Islands, (2) A local contribution, for example from the Izu-Mariana islands, falls short in explaining the origin of sediments of the Shikoku Basin as a whole, (3) The variation trend of these elements in the present samples is closely similar to those for the granites and volcanics. $\mathrm{SiO}_{2}, \mathrm{TiO}_{2}$ and $\mathrm{Al}_{2} \mathrm{O}_{3}$ have been cited as elements mutually stable during weathering; in addition, the relative concentrations of $\mathrm{Mg}, \mathrm{Ti}$, and $\mathrm{Fe}$ in the sediments were inherited from the source materials without remarkable modification.

Examination of trends for other elements leads us to the conclusion that $\mathrm{Si}, \mathrm{Ti}, \mathrm{Al}$, total $\mathrm{Fe}, \mathrm{Mg}$, and $\mathrm{K}$ behave as conservative elements, while $\mathrm{Ca}, \mathrm{Mn}$, and $\mathrm{FeO} / \mathrm{Fe}_{2} \mathrm{O}_{3}$ change remarkably during the sedimentary processes. $\mathrm{Fe}_{2} \mathrm{O}_{3}$ is negatively correlated with $\mathrm{FeO}$ (Figure 4) and positively correlated with total $\mathrm{Fe}$, indicating oxidation. 
TABLE 5

Chemical Composition (\%) of Argillaceous Sediments of Hole 444A

\begin{tabular}{|c|c|c|c|c|c|c|c|c|c|c|c|c|c|c|c|c|c|}
\hline No. & $\begin{array}{c}\text { Sample } \\
\text { (interval in } \mathrm{cm} \text { ) }\end{array}$ & $\mathrm{SiO}_{2}$ & $\mathrm{TiO}_{2}$ & $\mathrm{Al}_{2} \mathrm{O}_{3}$ & $\mathrm{Fe}_{2} \mathrm{O}_{3}$ & $\mathrm{FeO}$ & $\mathrm{MnO}$ & $\mathrm{MgO}$ & $\mathrm{CaO}$ & $\mathrm{Na}_{2} \mathrm{O}$ & $\mathrm{K}_{2} \mathrm{O}$ & $\mathrm{P}_{2} \mathrm{O}_{5}$ & $\mathrm{H}_{2} \mathrm{O}$ & $\mathrm{CaCO}_{3}$ & $\begin{array}{c}\text { Resid- } \\
\text { ual }^{*}\end{array}$ & Salt & Total \\
\hline 1 & $444 A-1-1,64-66$ & 57.04 & 0.70 & 17.71 & 4.86 & 1.93 & 0.24 & 2.95 & 0.59 & 1.62 & 3.03 & 0.17 & 6.01 & 2.43 & 0.14 & 1.59 & 101.01 \\
\hline 2 & $1-2,64-66$ & 55.68 & 0.68 & 15.82 & 4.68 & 1.94 & 0.21 & 2.51 & 2.32 & 1.83 & 2.46 & 0.15 & 6.01 & 2.57 & 0.43 & 1.81 & 99.10 \\
\hline 3 & $1-3,64-66$ & 39.79 & 0.53 & 12.02 & 4.05 & 1.11 & 0.32 & 1.69 & 0.09 & 1.08 & 2.56 & 0.20 & 3.73 & 29.22 & 0.53 & 2.07 & 99.00 \\
\hline 4 & $1-4,64-66$ & 54.89 & 0.69 & 16.19 & 4.68 & 1.74 & 0.34 & 2.60 & 0.94 & 1.24 & 2.92 & 0.16 & 8.27 & 2.44 & 0.30 & 2.88 & 100.28 \\
\hline 5 & $1-5,64-66$ & 58.88 & 0.67 & 17.03 & 4.76 & 1.85 & 0.21 & 2.66 & 1.49 & 1.44 & 2.78 & 0.15 & 5.59 & 1.30 & 0.21 & 2.13 & 101.16 \\
\hline 6 & $1-6,64-66$ & 56.87 & 0.66 & 16.17 & 4.88 & 1.71 & 0.29 & 2.40 & 2.14 & 1.86 & 2.74 & 0.16 & 5.95 & 0.0 & 1.70 & 1.50 & 99.03 \\
\hline 7 & $2-2,92-94$ & 61.40 & 0.47 & 12.51 & 2.75 & 1.54 & 0.15 & 1.31 & 2.06 & 2.08 & 1.88 & 0.084 & 5.70 & 4.88 & 1.09 & 1.32 & 99.23 \\
\hline 8 & $2-3,23-25$ & 59.61 & 0.59 & 15.91 & 4.02 & 1.94 & 0.11 & 2.31 & 1.95 & 1.39 & 2.93 & 0.12 & 5.75 & 0.0 & 0.89 & 1.51 & 99.04 \\
\hline 9 & $2-4,23-25$ & 56.07 & 0.68 & 16.19 & 4.90 & 1.98 & 0.33 & 2.53 & 2.68 & 2.40 & 2.51 & 0.18 & 6.91 & 0.0 & 1.43 & 1.36 & 100.14 \\
\hline 10 & $3-2,62-64$ & 55.77 & 0.71 & 16.55 & 5.11 & 1.63 & 0.35 & 2.90 & 2.50 & 1.98 & 2.53 & 0.17 & 6.85 & 0.0 & 0.18 & 1.88 & 99.11 \\
\hline 11 & $3-3,62-64$ & 55.35 & 0.74 & 16.21 & 4.76 & 2.20 & 0.33 & 2.87 & 2.79 & 2.15 & 2.32 & 0.19 & 4.89 & 0.0 & 2.87 & 1.34 & 99.01 \\
\hline 12 & $3-4,62-64$ & 55.49 & 0.71 & 16.57 & 5.32 & 1.71 & 0.42 & 2.97 & 1.17 & 1.83 & 2.62 & 0.18 & 5.42 & 2.24 & 0.64 & 1.59 & 98.88 \\
\hline 13 & $3-5,62-64$ & 57.58 & 0.71 & 16.43 & 5.04 & 1.67 & 0.39 & 2.77 & 2.42 & 2.26 & 2.56 & 0.17 & 6.61 & 0.0 & 0.23 & 1.52 & 100.37 \\
\hline 14 & $6-1,38-40$ & 57.71 & 0.65 & 17.13 & 5.93 & 1.04 & 0.36 & 2.94 & 1.96 & 1.82 & 2.73 & 0.18 & 5.69 & 0.0 & 1.11 & 1.78 & 101.03 \\
\hline 15 & $6-2,38-40$ & 55.14 & 0.65 & 16.95 & 5.78 & 1.11 & 0.35 & 2.55 & 1.85 & 1.73 & 2.66 & 0.19 & 7.04 & 0.0 & 1.34 & 1.69 & 99.02 \\
\hline 16 & $6-3,38-40$ & 54.85 & 0.69 & 16.31 & 6.30 & 1.11 & 0.49 & 2.79 & 1.86 & 1.94 & 2.68 & 0.14 & 7.81 & 0.0 & 0.32 & 1.75 & 99.04 \\
\hline 17 & $6-4,38-40$ & 58.08 & 0.68 & 17.01 & 5.46 & 1.01 & 0.39 & 2.61 & 1.52 & 2.16 & 2.95 & 0.18 & 6.00 & 0.0 & 1.33 & 1.53 & 100.92 \\
\hline 18 & $6-5,38-40$ & 57.14 & 0.67 & 17.18 & 5.56 & 1.28 & 0.32 & 2.69 & 2.14 & 1.97 & 2.65 & 0.16 & 6.91 & 0.0 & 0.32 & 1.37 & 100.36 \\
\hline 19 & $7-1,56-58$ & 56.19 & 0.70 & 16.81 & 6.22 & 1.11 & 0.41 & 3.05 & 2.09 & 1.92 & 2.70 & 0.17 & 7.34 & 0.0 & 0.00 & 1.42 & 100.14 \\
\hline 20 & $8-1,69-71$ & 56.80 & 0.71 & 17.09 & 6.24 & 1.19 & 0.30 & 3.01 & 1.70 & 1.66 & 2.58 & 0.14 & 6.82 & 0.0 & 0.75 & 1.78 & 100.76 \\
\hline 21 & $9-1,28-30$ & 55.55 & 0.66 & 15.61 & 5.77 & 1.75 & 0.42 & 2.54 & 3.22 & 0.12 & 2.07 & 0.14 & 6.05 & 0.0 & 0.02 & 5.24 & 99.15 \\
\hline 22 & $9-2,28-30$ & 54.93 & 0.72 & 15.57 & 5.73 & 2.57 & 0.57 & 3.54 & 4.50 & 2.86 & 1.78 & 0.16 & 6.55 & 0.0 & 0.05 & 1.43 & 100.97 \\
\hline 23 & $9-3,28-30$ & 54.40 & 0.71 & 15.08 & 5.70 & 2.67 & 0.53 & 3.60 & 4.08 & 2.45 & 1.82 & 0.14 & 6.06 & 0.0 & 0.48 & 1.39 & 99.12 \\
\hline 24 & $9-4,28-30$ & 55.94 & 0.74 & 15.08 & 5.10 & 3.11 & 0.30 & 3.10 & 3.17 & 2.29 & 1.61 & 0.54 & 5.87 & 2.35 & 0.57 & 1.21 & 100.99 \\
\hline 25 & $9-5,28-30$ & 54.92 & 0.72 & 15.21 & 5.27 & 3.31 & 0.26 & 3.27 & 4.53 & 2.20 & 1.72 & 0.14 & 5.99 & 0.0 & 0.05 & 1.46 & 99.04 \\
\hline 26 & $10-1,104-106$ & 52.03 & 0.76 & 13.93 & 6.19 & 3.33 & 0.22 & 3.14 & 4.61 & 1.29 & 1.34 & 0.12 & 4.56 & 6.84 & 0.41 & 1.78 & 100.55 \\
\hline 27 & $10-2,104-106$ & 55.38 & 0.65 & 13.44 & 3.54 & 2.91 & 0.19 & 2.11 & 3.50 & 2.21 & 1.24 & & & 8.85 & 0.45 & 1.12 & 100.25 \\
\hline 28 & $11-1,112-114$ & 65.73 & 0.42 & 12.89 & 1.94 & 1.62 & 0.14 & 1.44 & 2.58 & 2.42 & 2.04 & 0.097 & 4.87 & 0.0 & 1.88 & 0.98 & 99.05 \\
\hline 29 & $11-2,118-120$ & 59.40 & 0.63 & 15.38 & 3.77 & 2.38 & 0.19 & 2.19 & 3.77 & 2.02 & 1.83 & 0.14 & 4.66 & 0.0 & 1.47 & 1.23 & 99.06 \\
\hline 30 & $11-3,115-117$ & 59.46 & 0.40 & 11.43 & 2.07 & 1.60 & 0.15 & 1.29 & 0.78 & 2.12 & 1.72 & 0.081 & 4.03 & 11.92 & 0.32 & 1.69 & 99.06 \\
\hline 31 & $11-4,60-62$ & 54.62 & 0.56 & 14.24 & 3.88 & 2.69 & 0.21 & 2.39 & 3.29 & 1.61 & 1.92 & 0.14 & 5.79 & 4.74 & 1.37 & 1.88 & 99.33 \\
\hline 32 & $12-1,94-96$ & 46.52 & 0.63 & 13.11 & 4.27 & 2.78 & 0.26 & 2.79 & 0.56 & 1.68 & 1.93 & 0.12 & 2.77 & 19.54 & 1. & 1.70 & 99.91 \\
\hline 33 & $12-2,119-121$ & 52.27 & 0.66 & 14.23 & 5.47 & 2.44 & 0.17 & 3.03 & 4.10 & 1.79 & 1.77 & 0.14 & 5.03 & 5.74 & 1.00 & 2.10 & 99.93 \\
\hline 34 & $13-1,91-93$ & 54.77 & 0.74 & 15.30 & 5.79 & 3.46 & 0.17 & 3.36 & 5.72 & 1.51 & 1.40 & 0.13 & 4.83 & 0.0 & 0.35 & 1.64 & 99.18 \\
\hline 35 & $13-2,68-70$ & 55.37 & 0.73 & 15.69 & 5.70 & 2.84 & 0.31 & 2.92 & 3.95 & 1.44 & 1.98 & 0.13 & 5.56 & 0.0 & 0.53 & 1.77 & 98.93 \\
\hline 36 & $13-3,30-32$ & 55.95 & 0.70 & 15.17 & 5.56 & 2.78 & 0.16 & 2.98 & 3.36 & 1.53 & 2.17 & 0.13 & 5.79 & 0.0 & 1.32 & 1.46 & 99.05 \\
\hline 37 & $14-1,73-75$ & 56.39 & 0.66 & 14.87 & 4.51 & 2.73 & 0.24 & 2.80 & 2.34 & 1.63 & 2.03 & 0.15 & 5.81 & 2.53 & 0.92 & 1.86 & 99.47 \\
\hline 38 & $14-2,73-75$ & 62.56 & 0.58 & 13.26 & 2.57 & 2.73 & 0.14 & 1.95 & 3.47 & 2.28 & 1.53 & 0 . & 5.41 & 0.0 & 2 & 1.26 & 99.08 \\
\hline 39 & $14-3,73-75$ & 69.00 & 0.45 & 11.50 & 1.10 & 1.93 & 0.079 & 0.63 & 2.56 & 2.47 & 1.73 & 82 & 5.29 & 0.0 & 2.54 & 1.61 & 100.98 \\
\hline 40 & $14-4,26-28$ & 56.42 & 0.66 & 15.66 & 5.06 & 1.97 & 0.21 & 2.74 & 2.81 & 1.73 & 2.38 & 0.16 & 7.14 & 0.0 & 0.75 & 1.46 & 99.14 \\
\hline 41 & $15-1,44-46$ & 58.22 & 0.65 & 16.27 & 4.64 & 2.27 & 0.26 & 2.81 & 4.10 & 2.22 & 1.94 & 0.18 & 5.73 & 0.0 & 0.51 & 1.19 & 101.00 \\
\hline 42 & $15-2,44-46$ & 67.08 & 0.40 & 12.05 & 1. & 1.71 & 0.12 & 1. & 2. & 2. & 2.03 & 83 & 5.26 & 0. & 1.46 & 1.03 & 98.99 \\
\hline 43 & $15-3,44-46$ & 60.92 & 0.62 & 15.02 & 4.41 & 2.09 & 0.16 & 2.47 & 2.69 & 1.42 & 2.06 & 0.13 & 6.51 & 0.0 & 1 & 1.58 & 100.59 \\
\hline 44 & $16-1,44-46$ & 55.84 & 0.65 & 15.84 & 5.87 & 2.46 & 0.10 & 3.33 & 3.26 & 1.34 & 2.40 & & & 0.0 & 1.21 & 1.34 & 99.30 \\
\hline 45 & $16-3,74-76$ & 62.08 & 0.50 & 12.69 & 2.95 & 1.49 & 0.15 & 1.72 & 3.57 & 1.73 & 2.82 & 0.097 & & 0.0 & & 0.70 & 99.32 \\
\hline 46 & $21-1,122-12$ & 60.33 & 0.49 & 14.49 & 3.82 & 2.05 & 0.10 & 2.84 & 1.97 & 1.28 & 3.20 & 0.12 & 5.67 & 0.0 & 1.52 & 1.14 & 99.02 \\
\hline 47 & $21-2,33-35$ & 57.19 & 0.65 & 14.61 & 5.84 & 2.31 & 0.21 & 3.83 & 3.63 & 1.80 & 2.27 & 0.17 & & 0.0 & & 0.97 & 99.5 \\
\hline 48 & $21-2,35-37$ & 57.84 & 0.63 & 14.48 & 5.13 & 2.47 & 0.21 & 3.16 & 3.60 & 1.79 & 2.16 & & & 0.0 & 1.52 & 0.92 & 99.18 \\
\hline 49 & $21-2,39-41$ & 59.42 & 0.61 & 14.30 & 4.46 & 2.31 & 0.21 & 2.93 & 3.57 & 1.77 & 2.19 & 0.18 & & 0.0 & 0.67 & 0.83 & 99.02 \\
\hline 50 & $22-1,75-77$ & 61.30 & 0.61 & 13.25 & 2.67 & 2.88 & 0.20 & 2.00 & 3.13 & 2.79 & 2.31 & 0.12 & 4.19 & 0.0 & 2.77 & 0.82 & \\
\hline 51 & $22-2,75-77$ & 60.92 & 0.51 & 13.69 & 3.47 & 2.01 & 0.33 & 2.25 & 1.53 & 2.10 & 2.59 & 0.11 & 4.84 & 2.26 & 1.38 & 1.01 & 98.99 \\
\hline 52 & $22-3,95-97$ & 57.45 & 0.64 & 14.42 & 6.61 & 1.65 & 0.39 & 4.06 & 1.86 & 1.83 & 2.84 & 0.11 & 6.40 & 0.0 & 1.08 & 1.52 & 100.86 \\
\hline 53 & $22-3,99-101$ & 55.09 & 0.64 & 13.59 & 6.72 & 1.78 & 1.17 & 5.18 & 0.33 & 3.26 & 2.59 & 0.12 & & 3.68 & 1.41 & 1.28 & 100.95 \\
\hline 54 & $22-4,84-86$ & 51.82 & 0.60 & 13.29 & 5.50 & 1.77 & 0.52 & 4.10 & 2.15 & 1.87 & 2.48 & 0.11 & & & & 2.24 & \\
\hline 55 & $23-1,24-25$ & 47.79 & 0.50 & 11.24 & 4.88 & 1.39 & 0.54 & 1.95 & 0.21 & 2.73 & 2.22 & 0.20 & 3.51 & 20.00 & 1.44 & 0.94 & 99.55 \\
\hline
\end{tabular}

*Residual materials were calculated by subtracting $\mathrm{CO}_{2}$ and $\mathrm{H}_{2} \mathrm{O}$ from ignition loss. They may contain sulfur, organic matter, and other materials.

The emphasis in a majority of mineralogical and geochemical studies has been on the role of chemical and mechanical sorting during weathering and sedimentation. Nevertheless, the bulk chemical composition of sediments, except for some elements such as Mn and $\mathrm{Ca}$, may remain essentially unchanged over a vast region, although elements may migrate locally within the region.

\section{ACKNOWLEDGMENTS}

The author wishes to thank Dr. S. Mizutani, Nagoya University, and Dr. H. Okada, Shizuoka University, for their review of the manuscript. Thanks are also to Dr. K. Kobayashi, University of Tokyo, for providing valuable information. A part of the expenses of this study was defrayed by a Grant in Aid for Scientific Research of the Ministry of Education, Japan.

\section{REFERENCES}

Aramaki, S., Hirayama, K., and Nozawa, T., 1972. Chemical composition of Japanese granites, Part 2. Variation trends and average composition of 1200 analyses. J. Geol. Soc. Japan, 78, 39-49. 
TABLE 6

Chemical Composition (\%) Recalculated by Excluding Carbonates, Residual Materials, Water, and Salts, Hole 442A

\begin{tabular}{|c|c|c|c|c|c|c|c|c|c|c|c|c|c|}
\hline No. & $\mathrm{SiO}_{2}$ & $\mathrm{TiO}_{2}$ & $\mathrm{Al}_{2} \mathrm{O}_{3}$ & $\mathrm{Fe}_{2} \mathrm{O}_{3}$ & $\mathrm{FeO}$ & $\mathrm{MnO}$ & $\mathrm{MgO}$ & $\mathrm{CaO}$ & $\mathrm{Na}_{2} \mathrm{O}$ & $\mathrm{K}_{2} \mathrm{O}$ & $\mathrm{P}_{2} \mathrm{O}_{5}$ & $\mathrm{Fe}_{2} \mathrm{O}_{3} / \mathrm{FeO}$ & Total Fe \\
\hline 1 & 66.04 & 0.75 & 17.62 & 3.95 & 2.53 & 0.11 & 2.69 & 1.48 & 1.43 & 3.26 & 0.14 & 1.56 & 6.76 \\
\hline 2 & 66.50 & 0.74 & 17.34 & 4.10 & 2.21 & 0.12 & 2.48 & 1.66 & 1.54 & 3.14 & 0.15 & 1.85 & 6.56 \\
\hline 3 & 65.99 & 0.75 & 17.48 & 4.09 & 2.57 & 0.14 & 2.46 & 1.64 & 1.42 & 3.32 & 0.15 & 1.59 & 6.94 \\
\hline 4 & 65.91 & 0.78 & 18.19 & 3.76 & 2.68 & 0.17 & 2.63 & 0.97 & 1.08 & 3.70 & 0.13 & 1.40 & 6.74 \\
\hline 5 & 69.51 & 0.59 & 16.74 & 2.90 & 2.05 & 0.11 & 1.97 & 1.35 & 1.30 & 3.36 & 0.12 & 1.41 & 5.19 \\
\hline 6 & 66.26 & 0.74 & 17.58 & 3.87 & 2.53 & 0.13 & 2.70 & 1.59 & 1.04 & 3.45 & 0.12 & 1.52 & 6.68 \\
\hline 7 & 67.17 & 0.64 & 16.55 & 3.27 & 2.23 & 0.27 & 2.25 & 2.60 & 1.45 & 3.46 & 0.11 & 1.46 & 5.75 \\
\hline 8 & 66.11 & 0.75 & 17.63 & 3.80 & 2.42 & 0.32 & 2.91 & 1.04 & 1.30 & 3.60 & 0.13 & 1.57 & 6.49 \\
\hline 9 & 65.84 & 0.78 & 18.24 & 3.69 & 2.66 & 0.15 & 2.52 & 1.34 & 1.09 & 3.57 & 0.12 & 1.39 & 6.64 \\
\hline 10 & 65.50 & 0.77 & 17.94 & 3.54 & 2.70 & 0.23 & 2.70 & 1.55 & 1.38 & 3.55 & 0.13 & 1.31 & 6.55 \\
\hline 11 & 65.04 & 0.80 & 19.01 & 4.10 & 2.60 & 0.17 & 2.86 & 0.08 & 1.46 & 3.74 & 0.12 & 1.58 & 7.00 \\
\hline 12 & 66.89 & 0.78 & 17.81 & 4.04 & 2.53 & 0.31 & 2.85 & 0.65 & 1.64 & 2.35 & 0.15 & 1.60 & 6.84 \\
\hline 13 & 64.82 & 0.77 & 18.09 & 4.19 & 2.54 & 0.19 & 2.71 & 1.92 & 1.08 & 3.57 & 0.13 & 1.65 & 7.01 \\
\hline 14 & 65.56 & 0.77 & 17.65 & 4.34 & 2.40 & 0.19 & 2.78 & 1.29 & 1.31 & 3.57 & 0.13 & 1.81 & 7.01 \\
\hline 15 & 65.33 & 0.79 & 17.85 & 4.12 & 2.63 & 0.42 & 2.89 & 0.75 & 1.43 & 3.65 & 0.16 & 1.57 & 7.04 \\
\hline 16 & 65.86 & 0.80 & 18.05 & 4.46 & 2.51 & 0.25 & 2.88 & 0.70 & 0.53 & 3.83 & 0.14 & 1.78 & 7.25 \\
\hline 17 & 66.17 & 0.79 & 18.24 & 3.85 & 2.51 & 0.23 & 2.61 & 0.37 & 1.39 & 3.71 & 0.13 & 1.53 & 6.63 \\
\hline 18 & 65.38 & 0.74 & 18.46 & 3.79 & 2.33 & 0.25 & 2.65 & 1.42 & 1.49 & 3.37 & 0.14 & 1.63 & 6.38 \\
\hline 19 & 64.64 & 0.78 & 18.01 & 4.49 & 2.36 & 0.26 & 2.77 & 1.42 & 1.46 & 3.69 & 0.13 & 1.90 & 7.11 \\
\hline 20 & 66.91 & 0.73 & 17.49 & 4.09 & 2.28 & 0.41 & 2.56 & 0.16 & 1.65 & 3.62 & 0.11 & 1.80 & 6.62 \\
\hline 21 & 64.05 & 0.81 & 18.16 & 3.86 & 2.51 & 0.70 & 2.95 & 1.22 & 1.98 & 3.64 & 0.12 & 1.54 & 6.64 \\
\hline 22 & 66.03 & 0.81 & 18.93 & 3.50 & 2.54 & 0.20 & 2.87 & 0.16 & 1.14 & 3.73 & 0.11 & 1.38 & 6.32 \\
\hline 23 & 65.39 & 0.83 & 18.10 & 3.99 & 2.59 & 0.21 & 2.82 & 1.13 & 1.13 & 3.68 & 0.12 & 1.54 & 6.86 \\
\hline 24 & 66.75 & 0.75 & 17.45 & 3.80 & 2.42 & 0.49 & 2.69 & 0.11 & 1.65 & 3.75 & 0.13 & 1.57 & 6.49 \\
\hline 25 & 65.54 & 0.72 & 17.39 & 3.85 & 2.24 & 0.47 & 2.74 & 1.48 & 1.90 & 3.49 & 0.19 & 1.72 & 6.34 \\
\hline 26 & 65.68 & 0.77 & 18.21 & 4.03 & 2.36 & 0.27 & 2.84 & 0.38 & 1.68 & 3.65 & 0.12 & 1.71 & 6.66 \\
\hline 27 & 65.25 & 0.74 & 18.38 & 4.00 & 2.15 & 0.29 & 2.45 & 1.28 & 1.86 & 3.45 & 0.14 & 1.86 & 6.39 \\
\hline 28 & 65.52 & 0.75 & 17.53 & 4.06 & 2.38 & 0.35 & 2.66 & 1.40 & 1.71 & 3.51 & 0.12 & 1.70 & 6.71 \\
\hline 29 & 63.95 & 0.78 & 17.97 & 4.70 & 2.24 & 0.50 & 2.97 & 1.04 & 2.10 & 3.60 & 0.13 & 2.10 & 7.20 \\
\hline 30 & 65.19 & 0.77 & 18.42 & 3.73 & 2.43 & 0.42 & 2.97 & 0.02 & 2.35 & 3.58 & 0.13 & 1.54 & 6.43 \\
\hline 31 & 63.85 & 0.76 & 18.05 & 4.66 & 2.19 & 0.53 & 3.05 & 1.17 & 2.18 & 3.42 & 0.13 & 2.12 & 7.10 \\
\hline 32 & 70.48 & 0.53 & 15.89 & 2.66 & 1.77 & 0.12 & 1.85 & 1.21 & 1.82 & 3.58 & 0.091 & 1.51 & 4.62 \\
\hline 33 & 65.07 & 0.76 & 18.36 & 4.04 & 2.45 & 0.40 & 3.00 & 0.45 & 1.81 & 3.54 & 0.12 & 1.65 & 6.76 \\
\hline 34 & 63.85 & 0.75 & 18.50 & 4.25 & 2.26 & 0.45 & 2.92 & 1.27 & 2.16 & 3.46 & 0.14 & 1.88 & 6.76 \\
\hline 35 & 65.88 & 0.72 & 17.20 & 4.29 & 2.10 & 0.40 & 2.75 & 1.37 & 1.88 & 3.29 & 0.13 & 2.04 & 6.63 \\
\hline 36 & 62.87 & 0.78 & 18.51 & 4.85 & 2.36 & 0.46 & 3.24 & 1.23 & 1.99 & 3.59 & 0.13 & 2.05 & 7.47 \\
\hline 37 & 64.61 & 0.65 & 16.11 & 5.91 & 1.85 & 1.03 & 3.11 & 0.24 & 2.68 & 3.69 & 0.11 & 3.19 & 7.97 \\
\hline 38 & 63.46 & 0.80 & 19.13 & 4.06 & 2.45 & 0.27 & 3.10 & 1.11 & 1.66 & 3.84 & 0.12 & 1.66 & 6.79 \\
\hline 39 & 57.60 & 0.75 & 17.68 & 3.78 & 2.64 & 3.01 & 3.86 & 0.93 & 5.30 & 3.17 & 1.28 & 1.43 & 6.71 \\
\hline 40 & 62.81 & 0.84 & 19.31 & 5.06 & 2.19 & 0.32 & 2.97 & 1.20 & 1.67 & 3.50 & 0.13 & 2.31 & 7.50 \\
\hline 41 & 60.08 & 0.77 & 18.17 & 5.26 & 1.78 & 2.22 & 3.88 & 0.11 & 4.27 & 3.33 & 0.13 & 2.96 & 7.24 \\
\hline 42 & 63.05 & 0.80 & 19.46 & 5.38 & 1.90 & 0.13 & 2.91 & 1.15 & 1.76 & 3.33 & 0.12 & 2.82 & 7.50 \\
\hline 43 & 63.51 & 0.82 & 19.01 & 6.02 & 1.69 & 0.27 & 2.59 & 1.34 & 1.40 & 3.23 & 0.12 & 3.56 & 7.90 \\
\hline 44 & 63.79 & 0.82 & 19.63 & 5.28 & 1.84 & 0.12 & 2.61 & 0.97 & 1.26 & 3.55 & 0.13 & 2.88 & 7.32 \\
\hline 45 & 63.21 & 0.80 & 19.16 & 4.95 & 1.98 & 0.47 & 2.99 & 1.09 & 1.55 & 3.66 & 0.13 & 2.50 & 7.15 \\
\hline 46 & 64.17 & 0.76 & 18.23 & 5.41 & 1.67 & 0.23 & 2.86 & 1.48 & 1.71 & 3.35 & 0.14 & 3.23 & 7.27 \\
\hline 47 & 62.36 & 0.78 & 19.10 & 5.42 & 1.87 & 0.39 & 2.99 & 1.82 & 1.83 & 3.26 & 0.18 & 2.89 & 7.50 \\
\hline 48 & 62.12 & 0.82 & 18.47 & 5.68 & 1.57 & 1.00 & 2.87 & 1.70 & 2.26 & 3.31 & 0.21 & 3.61 & 7.42 \\
\hline 49 & 62.53 & 0.82 & 19.80 & 5.98 & 1.70 & 0.39 & 3.12 & 0.14 & 1.91 & 3.47 & 0.15 & 3.52 & 7.87 \\
\hline 50 & 62.51 & 0.80 & 19.40 & 5.81 & 1.65 & 0.56 & 3.06 & 0.60 & 1.97 & 3.47 & 0.16 & 3.52 & 7.65 \\
\hline 51 & 63.26 & 0.80 & 19.31 & 5.95 & 1.65 & 0.48 & 3.16 & 0.24 & 1.62 & 3.38 & 0.15 & 3.60 & 7.78 \\
\hline 52 & 61.83 & 0.79 & 19.46 & 5.97 & 1.53 & 0.80 & 3.10 & 0.77 & 2.28 & 3.28 & 0.19 & 3.91 & 7.67 \\
\hline 53 & 63.54 & 0.76 & 19.13 & 5.96 & 1.55 & 0.22 & 2.64 & 1.26 & 1.29 & 3.48 & 0.17 & 3.83 & 7.69 \\
\hline 54 & 62.90 & 0.78 & 19.36 & 6.01 & 1.58 & 0.14 & 2.87 & 1.27 & 1.54 & 3.43 & 0.13 & 3.81 & 7.76 \\
\hline 55 & 64.08 & 0.74 & 18.99 & 5.81 & 1.48 & 0.18 & 2.73 & 1.29 & 1.31 & 3.20 & 0.18 & 3.92 & 7.46 \\
\hline 56 & 62.96 & 0.76 & 18.86 & 6.89 & 0.54 & 0.79 & 2.67 & 1.02 & 1.93 & 3.43 & 0.15 & 12.79 & 7.49 \\
\hline 57 & 62.94 & 0.76 & 19.14 & 6.82 & 0.53 & 0.69 & 2.70 & 1.05 & 1.83 & 3.38 & 0.15 & 12.85 & 7.41 \\
\hline 58 & 63.23 & 0.77 & 19.16 & 6.06 & 1.17 & 0.34 & 2.89 & 0.94 & 1.74 & 3.52 & 0.18 & 5.18 & 7.37 \\
\hline 59 & 61.36 & 0.77 & 19.38 & 5.51 & 1.75 & 1.01 & 3.60 & 0.10 & 2.93 & 3.44 & 0.16 & 3.15 & 7.45 \\
\hline 60 & 61.49 & 0.78 & 18.84 & 5.89 & 1.42 & 1.12 & 3.51 & 0.20 & 3.25 & 3.33 & 0.18 & 4.16 & 7.47 \\
\hline 61 & 63.64 & 0.76 & 19.42 & 5.73 & 1.59 & 0.14 & 2.60 & 1.26 & 1.45 & 3.27 & 0.14 & 3.59 & 7.50 \\
\hline 62 & 63.08 & 0.78 & 19.24 & 6.23 & 1.40 & 0.17 & 2.89 & 1.35 & 1.39 & 3.33 & 0.15 & 4.43 & 7.79 \\
\hline
\end{tabular}


TABLE 6 - Continued

\begin{tabular}{rrrrrrrrrrrrrr}
\hline No. & $\mathrm{SiO}_{2}$ & $\mathrm{TiO}_{2}$ & $\mathrm{Al}_{2} \mathrm{O}_{3}$ & $\mathrm{Fe}_{2} \mathrm{O}_{3}$ & $\mathrm{FeO}$ & $\mathrm{MnO}$ & $\mathrm{MgO}$ & $\mathrm{CaO}$ & $\mathrm{Na}_{2} \mathrm{O}$ & $\mathrm{K}_{2} \mathrm{O}$ & $\mathrm{P}_{2} \mathrm{O}_{5}$ & $\mathrm{Fe}_{2} \mathrm{O}_{3} / \mathrm{FeO}$ & $\mathrm{Total} \mathrm{Fe}$ \\
\hline 63 & 63.30 & 0.72 & 18.06 & 5.43 & 1.22 & 0.54 & 2.69 & 1.78 & 2.77 & 3.29 & 0.20 & 4.44 & 6.79 \\
64 & 63.69 & 0.73 & 18.14 & 5.60 & 1.22 & 0.67 & 2.97 & 0.51 & 2.98 & 3.29 & 0.20 & 4.60 & 6.95 \\
65 & 62.92 & 0.71 & 18.80 & 5.90 & 0.87 & 0.51 & 2.65 & 1.84 & 2.52 & 3.10 & 0.19 & 6.80 & 6.86 \\
66 & 62.86 & 0.72 & 18.19 & 5.76 & 1.31 & 0.63 & 2.78 & 1.94 & 2.43 & 3.18 & 0.20 & 4.41 & 7.22 \\
67 & 63.62 & 0.77 & 18.36 & 6.02 & 1.68 & 0.18 & 2.23 & 2.49 & 1.73 & 2.78 & 0.15 & 3.59 & 7.89 \\
68 & 62.94 & 0.76 & 18.59 & 6.25 & 1.48 & 0.35 & 2.53 & 1.88 & 2.00 & 3.07 & 0.14 & 4.22 & 7.90 \\
69 & 62.11 & 0.75 & 18.16 & 6.06 & 1.64 & 1.32 & 3.28 & 0.19 & 3.27 & 3.07 & 0.14 & 3.69 & 7.89 \\
70 & 62.74 & 0.76 & 18.32 & 5.92 & 1.78 & 0.27 & 2.66 & 2.67 & 1.90 & 2.83 & 0.15 & 3.32 & 7.90 \\
71 & 63.16 & 0.72 & 17.81 & 6.32 & 1.56 & 0.59 & 3.47 & 0.94 & 2.53 & 2.73 & 0.17 & 4.05 & 8.06 \\
72 & 62.56 & 0.73 & 17.68 & 6.88 & 1.78 & 0.57 & 3.09 & 1.83 & 2.02 & 2.68 & 0.17 & 3.86 & 8.86 \\
73 & 63.18 & 0.72 & 17.85 & 7.03 & 1.64 & 0.46 & 2.90 & 1.11 & 2.08 & 2.87 & 0.15 & 4.28 & 8.86 \\
74 & 61.30 & 0.74 & 18.13 & 6.90 & 1.33 & 0.49 & 3.60 & 2.04 & 2.08 & 3.20 & 0.19 & 5.19 & 8.37 \\
75 & 60.88 & 0.70 & 16.56 & 9.81 & 0.18 & 0.32 & 4.26 & 2.52 & 2.37 & 2.12 & 0.27 & 53.95 & 10.01 \\
76 & 60.35 & 0.71 & 17.00 & 8.59 & 0.63 & 0.84 & 4.08 & 3.03 & 1.94 & 2.58 & 0.26 & 13.59 & 9.30 \\
77 & 59.56 & 0.74 & 16.62 & 9.46 & 0.09 & 0.95 & 4.63 & 2.52 & 2.62 & 2.57 & 0.22 & 106.39 & 9.56 \\
78 & 58.88 & 0.69 & 17.00 & 8.84 & 0.05 & 1.34 & 4.63 & 1.89 & 3.13 & 3.25 & 0.30 & 163.89 & 8.90 \\
79 & 58.56 & 0.69 & 16.52 & 10.80 & 0.05 & 1.92 & 4.93 & 1.86 & 0.60 & 3.50 & 0.59 & 235.14 & 10.85 \\
\hline
\end{tabular}

TABLE 7

Chemical Composition (\%) Recalculated by Excluding Carbonates, Residual Materials, Water, and Salts, Hole 442B

\begin{tabular}{rrrrrrrrrrrrrr}
\hline No. & $\mathrm{SiO}_{2}$ & $\mathrm{TiO}_{2}$ & $\mathrm{Al}_{2} \mathrm{O}_{3}$ & $\mathrm{Fe}_{2} \mathrm{O}_{3}$ & $\mathrm{FeO}$ & $\mathrm{MnO}$ & $\mathrm{MgO}$ & $\mathrm{CaO}$ & $\mathrm{Na}_{2} \mathrm{O}$ & $\mathrm{K}_{2} \mathrm{O}$ & $\mathrm{P}_{2} \mathrm{O}_{5}$ & $\frac{\mathrm{Fe}_{2} \mathrm{O}_{3}}{\mathrm{FeO}}$ & $\begin{array}{c}\mathrm{Total} \\
\mathrm{Fe}\end{array}$ \\
\hline 1 & 74.42 & 0.38 & 13.74 & 1.51 & 0.83 & 0.066 & 0.74 & 1.81 & 2.12 & 4.28 & 0.12 & 1.81 & 2.43 \\
2 & 63.30 & 0.74 & 17.88 & 6.32 & 1.58 & 0.030 & 3.22 & 2.46 & 1.30 & 3.00 & 0.18 & 4.00 & 8.07 \\
3 & 70.72 & 0.49 & 14.08 & 2.49 & 1.37 & 0.44 & 1.10 & 2.37 & 3.25 & 3.55 & 0.14 & 1.81 & 4.02 \\
4 & 58.14 & 0.70 & 15.79 & 10.43 & 0.04 & 1.42 & 4.53 & 2.40 & 3.73 & 2.43 & 0.39 & 283.56 & 10.47 \\
5 & 56.13 & 0.65 & 14.83 & 10.76 & 0.06 & 2.20 & 5.50 & 2.00 & 4.91 & 2.58 & 0.39 & 195.09 & 10.83 \\
6 & 57.30 & 0.65 & 15.55 & 9.61 & 0.13 & 1.77 & 5.84 & 2.12 & 4.11 & 2.57 & 0.35 & 71.47 & 9.76 \\
7 & 58.37 & 0.69 & 16.52 & 8.78 & 0.13 & 1.63 & 5.12 & 1.85 & 3.73 & 2.95 & 0.21 & 65.89 & 8.93 \\
8 & 57.88 & 0.67 & 15.93 & 9.72 & 0.12 & 1.80 & 4.81 & 1.76 & 3.82 & 3.18 & 0.31 & 81.80 & 9.85 \\
9 & 61.29 & 0.67 & 17.12 & 8.39 & 0.11 & 0.83 & 4.29 & 1.38 & 2.47 & 3.23 & 0.20 & 74.79 & 8.52 \\
10 & 54.47 & 0.56 & 12.74 & 12.43 & 0.44 & 3.89 & 4.59 & 1.36 & 6.09 & 3.17 & 0.26 & 28.26 & 12.92 \\
11 & 54.63 & 0.47 & 11.52 & 16.19 & 0.09 & 2.95 & 4.91 & 1.51 & 4.84 & 2.73 & 0.17 & 183.26 & 16.29 \\
12 & 56.95 & 0.54 & 13.31 & 11.04 & 0.09 & 2.99 & 6.82 & 1.08 & 4.51 & 2.50 & 0.18 & 122.76 & 11.14 \\
\hline
\end{tabular}

Boström, K., 1973. The origin and fate of ferromanganoan active ridge sediments. Acta Univ. Stockholmiensis, Stockholm Contr. Geol., 27, 149-243.

Boström, K., and Peterson, M. N. A., 1969. Origin of aluminium-poor ferromanganoan sediments in areas of high heat flow on the East Pacific Rise. Mar. Geol., 7, 427-447.

Boström, K., Joensuu, O., Valdes, S., Charm, W., and Glaccum, R., 1976. Geochemistry and origin of East Pacific sediment samples during DSDP Leg 34. In Yeats, R. S., et al., Init. Repts. DSDP, 34: Washington (U. S. Govt. Printing Office) pp. 559-574.

Geological Survey of Japan, 1960. Geology and Mineral Resources of Japan.

Goldberg, D. E., and Arrhenius, G. O. S., 1958. Chemistry of Pacific pelagic sediments. Geochim. Cosmochim Acta, 13, 153-212.

Karig, D. E., 1975. Basin genesis in the Philippine Sea. In Karig, D. E., Ingle, J. C., Jr., et al., Init. Repts. DSDP, 31: Washington (U. S. Govt. Printing Office), pp. 857-880.

Klein, G. D., Kobayashi, K., Chamley, H., Curtis, D. M., Dick, H. J. B., Echols, D. J., Fountain, D. M., Kinoshita, H., Marsh, N. G., Mizuno, A., Nisterenko, G. V., Okada, H., Sloan, J. R., Waples, D. M. and White, S. M., 1978. Off-ridge volcanism and seafloor spreading in the Shikoku Basin. Nature. 273, 746-748.
Klinkhammer, G., Bender, M.,' and Weiss, R. F., 1977. Hydrothermal manganese in the Galapagos Rift. Nature, 269, 319-320.

Kobayashi, K., and Nakada, M., 1978. Magnetic anomalies and tectonic evolution of the Shikoku Inter-Arc Basin. $J$. Phys. Earth, 26 (supplement), S391-S402.

Lupton, J. E., Weiss, R. F., and Carig, H., 1977. Mantle helium in hydrothermal plumes in the Galapagos Rift. Nature, 267, 603-604.

Murauchi, S., and Asanuma, T., 1977. Seismic Reflection Profiles in the Western Pacific, 1965-1974: Tokyo (Univ. of Tokyo Press).

Ono, C., 1976. Average chemical compositions of rocks and their graphic representation. 4. Mudstone of the Honshu geosyncline. Bull. Geol. Surv. Japan, 27, 519-533.

Rankama, K., and Sahama, H., 1950. Geochemistry: Chicago (Univ. Chicago Press).

Robertson, A. H. F., and Hudson, J. D., 1973. Cyprus umbers: chemical precipitates on a Tethyan ocean ridge. Earth Planet. Sci. Lett., 18, 93-101.

Sugisaki, R., 1976. Chemical characteristics of volcanic rocks: relation to plate movements. Lithos, 9, 17-30.

1978. Chemical composition of argillaceous sediments on the Pacific margin of southwest Japan. Cruise Repts. Geol. Surv. Japan, 9, 65-73. 
TABLE 8

Chemical Composition (\%) Recalculated by Excluding Carbonates, Residual Materials, Water, and Salts, Hole 443

\begin{tabular}{|c|c|c|c|c|c|c|c|c|c|c|c|c|c|}
\hline No. & $\mathrm{SiO}_{2}$ & $\mathrm{TiO}_{2}$ & $\mathrm{Al}_{2} \mathrm{O}_{3}$ & $\mathrm{Fe}_{2} \mathrm{O}_{3}$ & $\mathrm{FeO}$ & $\mathrm{MnO}$ & $\mathrm{MgO}$ & $\mathrm{CaO}$ & $\mathrm{Na}_{2} \mathrm{O}$ & $\mathrm{K}_{2} \mathrm{O}$ & $\mathrm{P}_{2} \mathrm{O}_{5}$ & $\mathrm{Fe}_{2} \mathrm{O}_{3} / \mathrm{FeO}$ & Total Fe \\
\hline 1 & 63.14 & 0.74 & 18.05 & 4.35 & 2.46 & 0.64 & 3.57 & 0.73 & 2.48 & 3.69 & 0.15 & 1.77 & 7.08 \\
\hline 2 & 65.80 & 0.74 & 18.13 & 3.79 & 2.60 & 0.33 & 2.71 & 0.45 & 1.82 & 3.47 & 0.17 & 1.46 & 6.67 \\
\hline 3 & 65.26 & 0.76 & 17.38 & 4.26 & 2.40 & 0.49 & 3.04 & 0.70 & 2.04 & 3.46 & 0.19 & 1.77 & 6.93 \\
\hline 4 & 66.50 & 0.81 & 16.33 & 4.03 & 2.72 & 0.18 & 3.46 & 0.18 & 1.81 & 3.85 & 0.14 & 1.48 & 7.05 \\
\hline 5 & 66.42 & 0.80 & 16.42 & 3.63 & 2.57 & 0.22 & 3.36 & 1.16 & 1.45 & 3.82 & 0.14 & 1.41 & 6.49 \\
\hline 6 & 68.01 & 0.80 & 16.57 & 3.32 & 2.66 & 0.18 & 3.26 & 0.15 & 1.15 & 3.79 & 0.12 & 1.25 & 6.27 \\
\hline 7 & 65.98 & 0.77 & 15.97 & 3.86 & 2.66 & 0.65 & 3.68 & 0.38 & 2.04 & 3.88 & 0.14 & 1.45 & 6.82 \\
\hline 8 & 67.62 & 0.76 & 16.04 & 3.94 & 2.48 & 0.50 & 3.06 & 0.02 & 1.64 & 3.81 & 0.14 & 1.59 & 6.69 \\
\hline 9 & 63.83 & 0.75 & 17.84 & 4.52 & 2.47 & 0.56 & 2.99 & 1.72 & 1.52 & 3.67 & 0.13 & 1.83 & 7.27 \\
\hline 10 & 67.24 & 0.78 & 18.24 & 3.33 & 2.62 & 0.19 & 2.60 & 0.16 & 1.20 & 3.50 & 0.14 & 1.27 & 6.25 \\
\hline 11 & 65.85 & 0.78 & 18.33 & 3.63 & 2.51 & 0.40 & 2.78 & 0.21 & 1.79 & 3.54 & 0.18 & 1.44 & 6.43 \\
\hline 12 & 64.56 & 0.81 & 18.47 & 3.95 & 2.61 & 0.28 & 2.99 & 0.46 & 2.00 & 3.72 & 0.15 & 1.51 & 6.85 \\
\hline 13 & 64.49 & 0.80 & 18.31 & 4.26 & 2.60 & 0.47 & 3.22 & 0.17 & 1.88 & 3.68 & 0.13 & 1.64 & 7.15 \\
\hline 14 & 64.57 & 0.78 & 17.94 & 4.44 & 2.57 & 0.42 & 3.26 & 0.08 & 2.16 & 3.64 & 0.15 & 1.73 & 7.30 \\
\hline 15 & 65.19 & 0.66 & 16.90 & 3.31 & 2.50 & 0.42 & 2.28 & 3.03 & 2.25 & 3.33 & 0.14 & 1.32 & 6.08 \\
\hline 16 & 66.64 & 0.69 & 17.86 & 3.47 & 2.34 & 0.41 & 2.37 & 0.30 & 2.43 & 3.33 & 0.15 & 1.48 & 6.08 \\
\hline 17 & 59.57 & 0.69 & 16.17 & 4.15 & 2.18 & 0.78 & 2.41 & 7.62 & 2.79 & 3.46 & 0.18 & 1.90 & 6.57 \\
\hline 18 & 67.90 & 0.68 & 17.27 & 2.84 & 2.21 & 0.12 & 2.10 & 1.40 & 1.99 & 3.39 & 0.11 & 1.28 & 5.30 \\
\hline 19 & 65.80 & 0.76 & 18.08 & 3.96 & 2.43 & 0.23 & 2.61 & 0.69 & 1.81 & 3.49 & 0.15 & 1.63 & 6.66 \\
\hline 20 & 65.19 & 0.79 & 18.22 & 4.36 & 2.76 & 0.53 & 2.82 & 0.03 & 1.33 & 3.78 & 0.19 & 1.58 & 7.42 \\
\hline 21 & 64.79 & 0.80 & 18.38 & 3.96 & 2.58 & 0.37 & 3.22 & 0.45 & 1.56 & 3.75 & 0.14 & 1.54 & 6.82 \\
\hline 22 & 64.76 & 0.81 & 18.60 & 4.61 & 2.42 & 0.30 & 3.00 & 0.33 & 1.28 & 3.69 & 0.21 & 1.91 & 7.29 \\
\hline 23 & 65.47 & 0.79 & 18.68 & 3.99 & 2.48 & 0.21 & 2.96 & 0.16 & 1.39 & 3.73 & 0.12 & 1.61 & 6.75 \\
\hline 24 & 65.24 & 0.75 & 18.91 & 4.42 & 2.21 & 0.31 & 2.74 & 0.14 & 1.71 & 3.42 & 0.14 & 2.00 & 6.88 \\
\hline 25 & 65.81 & 0.74 & 18.07 & 4.43 & 2.28 & 0.31 & 2.84 & 0.39 & 1.54 & 3.47 & 0.14 & 1.94 & 6.97 \\
\hline 26 & 66.47 & 0.69 & 16.60 & 4.22 & 2.10 & 0.30 & 2.76 & 1.81 & 1.69 & 3.24 & 0.12 & 2.01 & 6.55 \\
\hline 27 & 65.55 & 0.73 & 17.91 & 4.46 & 2.23 & 0.33 & 2.42 & 0.68 & 2.06 & 3.50 & 0.12 & 2.01 & 6.94 \\
\hline 28 & 64.48 & 0.72 & 17.82 & 4.82 & 2.32 & 0.30 & 2.88 & 1.55 & 1.53 & 3.45 & 0.12 & 2.07 & 7.40 \\
\hline 29 & 65.16 & 0.74 & 17.80 & 4.82 & 2.15 & 0.27 & 2.58 & 0.77 & 2.11 & 3.47 & 0.15 & 2.24 & 7.21 \\
\hline 30 & 65.08 & 0.75 & 18.37 & 4.42 & 2.42 & 0.30 & 3.06 & 0.20 & 1.71 & 3.55 & 0.14 & 1.82 & 7.11 \\
\hline 31 & 64.24 & 0.76 & 18.35 & 4.82 & 2.26 & 0.34 & 3.09 & 0.46 & 1.91 & 3.65 & 0.13 & 2.13 & 7.33 \\
\hline 32 & 64.88 & 0.79 & 17.61 & 4.71 & 2.34 & 0.50 & 3.15 & 0.21 & 2.03 & 3.63 & 0.14 & 2.01 & 7.31 \\
\hline 33 & 67.38 & 0.61 & 16.68 & 4.87 & 2.06 & 0.14 & 2.62 & 0.08 & 1.54 & 3.92 & 0.093 & 2.37 & 7.16 \\
\hline 34 & 64.23 & 0.78 & 18.05 & 4.22 & 2.48 & 0.44 & 2.81 & 0.57 & 2.44 & 3.83 & 0.16 & 1.70 & 6.97 \\
\hline 35 & 63.70 & 0.76 & 17.70 & 4.73 & 2.49 & 0.72 & 3.14 & 0.23 & 2.75 & 3.57 & 0.20 & 1.90 & 7.50 \\
\hline 36 & 65.89 & 0.77 & 18.14 & 4.03 & 2.36 & 0.19 & 2.94 & 0.24 & 1.73 & 3.59 & 0.12 & 1.71 & 6.65 \\
\hline 37 & 65.78 & 0.72 & 17.90 & 3.95 & 2.29 & 0.43 & 2.76 & 0.22 & 2.06 & 3.77 & 0.12 & 1.72 & 6.50 \\
\hline 38 & 65.20 & 0.78 & 17.96 & 3.91 & 2.47 & 0.15 & 2.78 & 1.43 & 1.78 & 3.42 & 0.12 & 1.59 & 6.65 \\
\hline 39 & 63.49 & 0.78 & 18.61 & 4.59 & 2.49 & 0.33 & 2.85 & 1.19 & 1.93 & 3.61 & 0.12 & 1.85 & 7.36 \\
\hline 40 & 65.36 & 0.72 & 18.02 & 4.50 & 2.21 & 0.099 & 2.44 & 1.35 & 1.53 & 3.65 & 0.11 & 2.03 & 6.95 \\
\hline 41 & 66.21 & 0.78 & 18.32 & 3.69 & 2.53 & 0.18 & 2.35 & 0.61 & 1.74 & 3.46 & 0.13 & 1.46 & 6.50 \\
\hline 42 & 65.03 & 0.83 & 19.46 & 3.50 & 2.48 & 0.15 & 2.67 & 0.30 & 1.72 & 3.71 & 0.14 & 1.41 & 6.26 \\
\hline 43 & 62.86 & 0.78 & 18.57 & 4.99 & 2.45 & 0.67 & 3.24 & 0.41 & 2.18 & 3.71 & 0.15 & 2.04 & 7.70 \\
\hline 44 & 63.86 & 0.80 & 19.08 & 4.59 & 2.40 & 0.34 & 3.12 & 0.27 & 1.70 & 3.72 & 0.13 & 1.91 & 7.25 \\
\hline 45 & 64.57 & 0.80 & 18.98 & 4.56 & 2.43 & 0.32 & 2.99 & 0.34 & 1.30 & 3.57 & 0.15 & 1.88 & 7.26 \\
\hline 46 & 63.61 & 0.84 & 19.21 & 4.55 & 2.54 & 0.54 & 2.97 & 0.19 & 1.62 & 3.79 & 0.13 & 1.79 & 7.37 \\
\hline 47 & 63.24 & 0.77 & 18.23 & 4.94 & 2.29 & 0.53 & 3.16 & 1.18 & 2.10 & 3.43 & 0.14 & 2.16 & 7.48 \\
\hline 48 & 66.95 & 0.69 & 18.35 & 3.91 & 2.13 & 0.16 & 2.27 & 0.21 & 1.48 & 3.76 & 0.11 & 1.84 & 6.27 \\
\hline 49 & 71.89 & 0.47 & 15.62 & 2.31 & 1.65 & 0.19 & 1.28 & 1.35 & 2.10 & 3.06 & 0.093 & 1.41 & 4.14 \\
\hline 50 & 62.81 & 0.81 & 19.27 & 5.27 & 1.96 & 0.62 & 3.33 & 0.27 & 1.93 & 3.60 & 0.14 & 2.69 & 7.45 \\
\hline 51 & 62.65 & 0.77 & 18.48 & 5.69 & 1.64 & 0.40 & 3.10 & 0.28 & 3.31 & 3.55 & 0.13 & 3.48 & 7.51 \\
\hline 52 & 64.80 & 0.69 & 17.65 & 4.52 & 1.88 & 0.38 & 2.71 & 1.72 & 2.01 & 3.50 & 0.14 & 2.41 & 6.60 \\
\hline 53 & 67.61 & 0.57 & 17.75 & 4.17 & 1.48 & 0.37 & 1.97 & 0.35 & 1.82 & 3.78 & 0.12 & 2.82 & 5.82 \\
\hline 54 & 67.13 & 0.60 & 17.72 & 4.42 & 1.55 & 0.37 & 2.37 & 0.21 & 1.77 & 3.75 & 0.11 & 2.86 & 6.14 \\
\hline 55 & 62.49 & 0.77 & 18.61 & 5.82 & 1.86 & 0.57 & 3.53 & 0.97 & 1.95 & 3.24 & 0.17 & 3.12 & 7.89 \\
\hline 56 & 63.21 & 0.81 & 19.20 & 5.60 & 1.92 & 0.32 & 3.33 & 0.56 & 1.40 & 3.49 & 0.16 & 2.91 & 7.74 \\
\hline 57 & 63.25 & 0.81 & 18.44 & 5.32 & 2.19 & 0.42 & 3.02 & 1.40 & 1.86 & 3.13 & 0.15 & 2.43 & 7.75 \\
\hline 58 & 62.74 & 0.78 & 18.82 & 5.83 & 2.01 & 0.44 & 3.40 & 0.49 & 2.14 & 3.19 & 0.16 & 2.90 & 8.06 \\
\hline 59 & 61.62 & 0.80 & 17.84 & 6.59 & 2.92 & 0.28 & 3.46 & 2.35 & 1.43 & 2.57 & 0.15 & 2.26 & 9.83 \\
\hline 60 & 62.46 & 0.82 & 18.71 & 6.35 & 1.86 & 0.82 & 3.29 & 0.41 & 1.70 & 3.39 & 0.20 & 3.41 & 8.42 \\
\hline
\end{tabular}


TABLE 8 - Continued

\begin{tabular}{|c|c|c|c|c|c|c|c|c|c|c|c|c|c|}
\hline No. & $\mathrm{SiO}_{2}$ & $\mathrm{TiO}_{2}$ & $\mathrm{Al}_{2} \mathrm{O}_{3}$ & $\mathrm{Fe}_{2} \mathrm{O}_{3}$ & $\mathrm{FeO}$ & $\mathrm{MnO}$ & $\mathrm{MgO}$ & $\mathrm{CaO}$ & $\mathrm{Na}_{2} \mathrm{O}$ & $\mathrm{K}_{2} \mathrm{O}$ & $\mathrm{P}_{2} \mathrm{O}_{5}$ & $\mathrm{Fe}_{2} \mathrm{O}_{3} / \mathrm{FeO}$ & Total Fe \\
\hline $\begin{array}{l}61 \\
62 \\
63 \\
64 \\
65\end{array}$ & $\begin{array}{l}59.27 \\
61.39 \\
62.91 \\
62.86 \\
63.29\end{array}$ & $\begin{array}{l}1.01 \\
0.77 \\
0.77 \\
0.74 \\
0.81\end{array}$ & $\begin{array}{l}16.90 \\
18.07 \\
18.17 \\
17.90 \\
17.92\end{array}$ & $\begin{array}{l}6.09 \\
5.69 \\
5.57 \\
5.28 \\
6.06\end{array}$ & $\begin{array}{l}5.20 \\
1.95 \\
2.32 \\
2.43 \\
2.61\end{array}$ & $\begin{array}{l}0.26 \\
0.71 \\
0.36 \\
0.42 \\
0.61\end{array}$ & $\begin{array}{l}3.57 \\
3.20 \\
3.04 \\
2.78 \\
3.20\end{array}$ & $\begin{array}{l}5.33 \\
0.40 \\
2.56 \\
2.84 \\
0.17\end{array}$ & $\begin{array}{l}1.04 \\
2.46 \\
1.44 \\
1.83 \\
2.25\end{array}$ & $\begin{array}{l}1.23 \\
3.17 \\
2.73 \\
2.77 \\
2.93\end{array}$ & $\begin{array}{l}0.11 \\
2.19 \\
0.13 \\
0.14 \\
0.15\end{array}$ & $\begin{array}{l}1.17 \\
2.92 \\
2.40 \\
2.17 \\
2.32\end{array}$ & $\begin{array}{r}11.87 \\
7.86 \\
8.15 \\
7.99 \\
8.97\end{array}$ \\
\hline $\begin{array}{l}66 \\
67 \\
68 \\
69 \\
70\end{array}$ & $\begin{array}{l}67.73 \\
62.96 \\
65.27 \\
63.78 \\
65.35\end{array}$ & $\begin{array}{l}0.65 \\
0.74 \\
0.67 \\
0.78 \\
0.75\end{array}$ & $\begin{array}{l}16.71 \\
17.80 \\
17.99 \\
18.77 \\
18.64\end{array}$ & $\begin{array}{l}4.27 \\
5.95 \\
1.77 \\
5.67 \\
5.56\end{array}$ & $\begin{array}{l}2.18 \\
1.69 \\
1.60 \\
1.70 \\
1.56\end{array}$ & $\begin{array}{l}0.31 \\
0.94 \\
1.51 \\
0.29 \\
0.11\end{array}$ & $\begin{array}{l}2.36 \\
3.29 \\
3.37 \\
2.79 \\
2.39\end{array}$ & $\begin{array}{l}0.70 \\
0.60 \\
0.13 \\
1.36 \\
1.04\end{array}$ & $\begin{array}{l}2.32 \\
2.59 \\
4.12 \\
1.26 \\
1.13\end{array}$ & $\begin{array}{l}2.63 \\
3.24 \\
3.33 \\
3.44 \\
3.34\end{array}$ & $\begin{array}{l}0.14 \\
0.18 \\
0.24 \\
0.17 \\
0.14\end{array}$ & $\begin{array}{l}1.96 \\
3.51 \\
1.11 \\
3.34 \\
3.56\end{array}$ & $\begin{array}{l}6.69 \\
7.83 \\
3.54 \\
7.56 \\
7.29\end{array}$ \\
\hline $\begin{array}{l}71 \\
72 \\
73 \\
74 \\
75\end{array}$ & $\begin{array}{l}64.06 \\
64.65 \\
63.43 \\
61.72 \\
63.07\end{array}$ & $\begin{array}{l}0.74 \\
0.73 \\
0.75 \\
0.73 \\
0.66\end{array}$ & $\begin{array}{l}18.60 \\
18.73 \\
19.22 \\
18.40 \\
18.56\end{array}$ & $\begin{array}{l}5.62 \\
5.36 \\
5.68 \\
4.97 \\
5.41\end{array}$ & $\begin{array}{l}1.52 \\
1.65 \\
1.60 \\
1.74 \\
1.86\end{array}$ & $\begin{array}{l}0.32 \\
0.49 \\
0.41 \\
0.43 \\
0.42\end{array}$ & $\begin{array}{l}2.77 \\
2.78 \\
2.94 \\
2.81 \\
2.88\end{array}$ & $\begin{array}{l}1.13 \\
0.21 \\
1.24 \\
0.54 \\
1.65\end{array}$ & $\begin{array}{l}1.67 \\
2.05 \\
1.20 \\
5.20 \\
2.07\end{array}$ & $\begin{array}{l}3.43 \\
3.20 \\
3.40 \\
3.31 \\
3.25\end{array}$ & $\begin{array}{l}0.15 \\
0.15 \\
0.13 \\
0.15 \\
0.16\end{array}$ & $\begin{array}{l}3.71 \\
3.25 \\
3.54 \\
2.85 \\
2.91\end{array}$ & $\begin{array}{l}7.30 \\
7.20 \\
7.46 \\
6.91 \\
7.48\end{array}$ \\
\hline $\begin{array}{l}76 \\
77 \\
78 \\
79 \\
80\end{array}$ & $\begin{array}{l}63.48 \\
63.88 \\
63.76 \\
62.70 \\
63.61\end{array}$ & $\begin{array}{l}0.75 \\
0.70 \\
0.73 \\
0.73 \\
0.74\end{array}$ & $\begin{array}{l}18.64 \\
17.45 \\
17.64 \\
17.56 \\
17.18\end{array}$ & $\begin{array}{l}6.00 \\
5.19 \\
5.60 \\
5.40 \\
5.42\end{array}$ & $\begin{array}{l}1.70 \\
1.81 \\
2.06 \\
2.29 \\
2.62\end{array}$ & $\begin{array}{l}0.60 \\
0.75 \\
0.14 \\
0.52 \\
0.50\end{array}$ & $\begin{array}{l}3.05 \\
3.43 \\
2.69 \\
3.12 \\
3.24\end{array}$ & $\begin{array}{l}0.09 \\
0.87 \\
2.72 \\
2.08 \\
1.62\end{array}$ & $\begin{array}{l}2.32 \\
2.67 \\
1.82 \\
2.96 \\
2.21\end{array}$ & $\begin{array}{l}3.21 \\
3.07 \\
2.69 \\
2.51 \\
2.71\end{array}$ & $\begin{array}{l}0.16 \\
0.18 \\
0.14 \\
0.15 \\
0.16\end{array}$ & $\begin{array}{l}3.52 \\
2.86 \\
2.72 \\
2.36 \\
2.07\end{array}$ & $\begin{array}{l}7.90 \\
7.20 \\
7.89 \\
7.94 \\
8.33\end{array}$ \\
\hline $\begin{array}{l}81 \\
82 \\
83 \\
84 \\
85\end{array}$ & $\begin{array}{l}63.24 \\
65.48 \\
61.97 \\
62.85 \\
68.85\end{array}$ & $\begin{array}{l}0.73 \\
0.69 \\
0.77 \\
0.91 \\
0.44\end{array}$ & $\begin{array}{l}17.21 \\
16.64 \\
17.62 \\
17.44 \\
14.90\end{array}$ & $\begin{array}{l}5.48 \\
5.40 \\
5.54 \\
5.65 \\
3.13\end{array}$ & $\begin{array}{l}2.18 \\
2.22 \\
2.69 \\
2.71 \\
1.79\end{array}$ & $\begin{array}{l}0.60 \\
0.52 \\
0.60 \\
0.30 \\
0.24\end{array}$ & $\begin{array}{l}3.15 \\
2.80 \\
2.86 \\
2.77 \\
1.48\end{array}$ & $\begin{array}{l}1.92 \\
1.13 \\
2.65 \\
2.10 \\
2.62\end{array}$ & $\begin{array}{l}2.55 \\
2.22 \\
2.76 \\
2.58 \\
2.75\end{array}$ & $\begin{array}{l}2.80 \\
2.76 \\
2.41 \\
2.50 \\
3.70\end{array}$ & $\begin{array}{l}0.16 \\
0.13 \\
0.14 \\
0.19 \\
0.094\end{array}$ & $\begin{array}{l}2.52 \\
2.43 \\
2.06 \\
2.08 \\
1.75\end{array}$ & $\begin{array}{l}7.90 \\
7.87 \\
8.53 \\
8.66 \\
5.13\end{array}$ \\
\hline $\begin{array}{l}86 \\
87 \\
88 \\
89 \\
90\end{array}$ & $\begin{array}{l}63.34 \\
65.73 \\
62.72 \\
65.39 \\
65.00\end{array}$ & $\begin{array}{l}0.78 \\
0.65 \\
0.73 \\
0.69 \\
0.75\end{array}$ & $\begin{array}{l}17.18 \\
16.53 \\
17.25 \\
17.25 \\
17.29\end{array}$ & $\begin{array}{l}5.72 \\
5.21 \\
6.25 \\
5.25 \\
4.84\end{array}$ & $\begin{array}{l}2.34 \\
1.95 \\
2.01 \\
1.81 \\
2.40\end{array}$ & $\begin{array}{l}0.43 \\
0.30 \\
0.52 \\
0.53 \\
0.19\end{array}$ & $\begin{array}{l}2.59 \\
2.66 \\
2.91 \\
2.21 \\
2.71\end{array}$ & $\begin{array}{l}2.23 \\
1.93 \\
1.99 \\
1.26 \\
2.13\end{array}$ & $\begin{array}{l}2.53 \\
2.23 \\
2.63 \\
2.51 \\
2.18\end{array}$ & $\begin{array}{l}2.71 \\
2.68 \\
2.81 \\
2.94 \\
2.38\end{array}$ & $\begin{array}{l}0.16 \\
0.13 \\
0.18 \\
0.16 \\
0.13\end{array}$ & $\begin{array}{l}2.45 \\
2.67 \\
3.10 \\
2.90 \\
2.02\end{array}$ & $\begin{array}{l}8.32 \\
7.37 \\
8.48 \\
7.27 \\
7.51\end{array}$ \\
\hline $\begin{array}{l}91 \\
92 \\
93 \\
94 \\
95\end{array}$ & $\begin{array}{l}66.14 \\
63.62 \\
60.52 \\
57.07 \\
53.73\end{array}$ & $\begin{array}{l}0.60 \\
0.77 \\
0.78 \\
0.75 \\
0.69\end{array}$ & $\begin{array}{l}16.11 \\
17.16 \\
16.72 \\
16.04 \\
15.37\end{array}$ & $\begin{array}{l}5.15 \\
6.64 \\
6.21 \\
6.11 \\
6.41\end{array}$ & $\begin{array}{l}2.25 \\
2.39 \\
2.44 \\
2.41 \\
1.82\end{array}$ & $\begin{array}{l}0.50 \\
0.47 \\
0.38 \\
0.48 \\
2.06\end{array}$ & $\begin{array}{l}2.25 \\
2.96 \\
2.48 \\
2.82 \\
3.48\end{array}$ & $\begin{array}{r}0.69 \\
1.09 \\
5.78 \\
9.23 \\
10.05\end{array}$ & $\begin{array}{l}2.92 \\
1.97 \\
1.96 \\
2.31 \\
3.51\end{array}$ & $\begin{array}{l}3.26 \\
2.77 \\
2.60 \\
2.61 \\
2.74\end{array}$ & $\begin{array}{l}0.14 \\
0.16 \\
0.13 \\
0.16 \\
0.13\end{array}$ & $\begin{array}{l}2.29 \\
2.78 \\
2.55 \\
2.53 \\
3.52\end{array}$ & $\begin{array}{l}7.65 \\
9.29 \\
8.92 \\
8.80 \\
8.43\end{array}$ \\
\hline $\begin{array}{r}96 \\
97 \\
98 \\
99 \\
100\end{array}$ & $\begin{array}{l}61.34 \\
61.94 \\
63.96 \\
63.33 \\
55.02\end{array}$ & $\begin{array}{l}0.72 \\
0.76 \\
0.79 \\
0.77 \\
0.66\end{array}$ & $\begin{array}{l}17.72 \\
16.80 \\
17.92 \\
17.56 \\
14.14\end{array}$ & $\begin{array}{l}5.95 \\
6.41 \\
5.89 \\
5.85 \\
5.17\end{array}$ & $\begin{array}{l}1.96 \\
2.12 \\
2.39 \\
2.19 \\
1.92\end{array}$ & $\begin{array}{l}0.13 \\
1.62 \\
0.23 \\
0.61 \\
4.16\end{array}$ & $\begin{array}{l}4.55 \\
3.41 \\
2.98 \\
3.54 \\
3.36\end{array}$ & $\begin{array}{l}2.94 \\
0.90 \\
0.90 \\
0.23 \\
6.10\end{array}$ & $\begin{array}{l}1.57 \\
2.73 \\
1.85 \\
2.48 \\
5.32\end{array}$ & $\begin{array}{l}3.01 \\
3.18 \\
3.00 \\
3.31 \\
2.60\end{array}$ & $\begin{array}{l}0.091 \\
0.13 \\
0.10 \\
0.13 \\
1.54\end{array}$ & $\begin{array}{l}3.03 \\
3.03 \\
2.46 \\
2.68 \\
2.69\end{array}$ & $\begin{array}{l}8.13 \\
8.77 \\
8.55 \\
8.28 \\
7.30\end{array}$ \\
\hline $\begin{array}{l}101 \\
102 \\
103 \\
104 \\
105\end{array}$ & $\begin{array}{l}61.91 \\
62.65 \\
62.17 \\
63.81 \\
65.26\end{array}$ & $\begin{array}{l}0.75 \\
0.74 \\
0.69 \\
0.73 \\
0.66\end{array}$ & $\begin{array}{l}17.86 \\
17.65 \\
16.88 \\
17.31 \\
16.63\end{array}$ & $\begin{array}{l}6.93 \\
6.56 \\
6.76 \\
6.26 \\
5.34\end{array}$ & $\begin{array}{l}1.82 \\
1.81 \\
1.60 \\
2.12 \\
1.80\end{array}$ & $\begin{array}{l}0.19 \\
0.40 \\
0.67 \\
0.56 \\
0.22\end{array}$ & $\begin{array}{l}3.16 \\
3.19 \\
3.55 \\
3.07 \\
2.92\end{array}$ & $\begin{array}{l}2.47 \\
2.45 \\
1.72 \\
0.47 \\
2.06\end{array}$ & $\begin{array}{l}1.74 \\
1.41 \\
2.51 \\
2.27 \\
1.77\end{array}$ & $\begin{array}{l}3.02 \\
3.00 \\
3.34 \\
3.26 \\
3.22\end{array}$ & $\begin{array}{l}0.14 \\
0.15 \\
0.12 \\
0.14 \\
0.12\end{array}$ & $\begin{array}{l}3.82 \\
3.63 \\
4.24 \\
2.95 \\
2.96\end{array}$ & $\begin{array}{l}8.95 \\
8.57 \\
8.53 \\
8.62 \\
7.34\end{array}$ \\
\hline $\begin{array}{l}106 \\
107 \\
108 \\
109 \\
110\end{array}$ & $\begin{array}{l}63.78 \\
63.89 \\
66.02 \\
62.43 \\
62.50\end{array}$ & $\begin{array}{l}0.66 \\
0.73 \\
0.63 \\
0.81 \\
0.73\end{array}$ & $\begin{array}{l}15.73 \\
16.51 \\
15.83 \\
15.78 \\
16.26\end{array}$ & $\begin{array}{l}4.66 \\
6.08 \\
4.93 \\
7.61 \\
6.86\end{array}$ & $\begin{array}{l}2.29 \\
2.05 \\
2.25 \\
2.20 \\
2.00\end{array}$ & $\begin{array}{l}2.45 \\
0.56 \\
0.10 \\
1.06 \\
0.83\end{array}$ & $\begin{array}{l}2.51 \\
3.33 \\
2.39 \\
3.50 \\
3.51\end{array}$ & $\begin{array}{l}2.74 \\
1.30 \\
2.89 \\
0.85 \\
1.20\end{array}$ & $\begin{array}{l}1.89 \\
2.51 \\
1.83 \\
2.82 \\
3.32\end{array}$ & $\begin{array}{l}3.10 \\
2.90 \\
3.03 \\
2.67 \\
2.67\end{array}$ & $\begin{array}{l}0.21 \\
0.16 \\
0.12 \\
0.26 \\
0.13\end{array}$ & $\begin{array}{l}2.03 \\
2.97 \\
2.19 \\
3.46 \\
3.43\end{array}$ & $\begin{array}{r}7.20 \\
8.35 \\
7.42 \\
10.06 \\
9.08\end{array}$ \\
\hline $\begin{array}{l}111 \\
112 \\
113 \\
114\end{array}$ & $\begin{array}{l}63.94 \\
65.14 \\
62.51 \\
55.74\end{array}$ & $\begin{array}{l}0.68 \\
0.70 \\
0.65 \\
0.70\end{array}$ & $\begin{array}{l}16.44 \\
16.28 \\
15.58 \\
15.01\end{array}$ & $\begin{array}{r}6.71 \\
7.07 \\
10.37 \\
10.85\end{array}$ & $\begin{array}{l}1.92 \\
1.98 \\
0.62 \\
0.47\end{array}$ & $\begin{array}{l}0.14 \\
0.75 \\
0.58 \\
1.13\end{array}$ & $\begin{array}{l}3.17 \\
2.57 \\
4.61 \\
5.08\end{array}$ & $\begin{array}{l}2.37 \\
0.10 \\
0.50 \\
7.01\end{array}$ & $\begin{array}{l}1.68 \\
2.12 \\
1.43 \\
0.92\end{array}$ & $\begin{array}{l}2.84 \\
3.04 \\
3.02 \\
2.87\end{array}$ & $\begin{array}{l}0.11 \\
0.24 \\
0.13 \\
0.22\end{array}$ & $\begin{array}{r}3.49 \\
3.57 \\
16.63 \\
23.29\end{array}$ & $\begin{array}{r}8.84 \\
9.27 \\
11.07 \\
11.37 \\
\end{array}$ \\
\hline
\end{tabular}

1979. Chemical composition of argillaceous sediments around the Yamato Bank in the Japan Sea. Cruise Repts. Geol. Surv. Japan, 13, 75-88.

in press a. Chemical composition of argillaceous sediments around the Nishitsugaru Basin in the Japan Sea. Cruise Repts. Geol. Surv. Japan.

in press b. Major element chemistry of the Japan

Trench. In Langseth, M., et al., Init. Repts DSDP, 56, 57: Washington (U. S. Govt. Printing Office).
Sugisaki, R., and Honza, E., in press. Chemical composition of argillaceous sediments in the Pacific margin of northeast Japan. Bull. Geol. Surv. Japan.

Sugisaki, R., and Kinoshita, T., in press. Chemical composition of argillaceous sediments around the Oki Islands, the Japan Sea. Cruise Repts. Geol. Surv. Japan.

Sugisaki, R., Suzuki, T., Kanmera, K., Sakai, T., and Sano, H., 1979. Chemical composition of green rocks in the Shimanto Belt, southwest Japan. J. Geol. Soc. Japan, 85, 455-466. 
TABLE 9

Chemical Composition (\%) Recalculated by Excluding Carbonates, Residual Materials, Water, and Salts, Hole 444

\begin{tabular}{rrllllllllllll}
\hline No. & $\mathrm{SiO}_{2}$ & $\mathrm{TiO}_{2}$ & $\mathrm{Al}_{2} \mathrm{O}_{3}$ & $\mathrm{Fe}_{2} \mathrm{O}_{3}$ & $\mathrm{FeO}$ & $\mathrm{MnO}$ & $\mathrm{MgO}$ & $\mathrm{CaO}$ & $\mathrm{Na}_{2} \mathrm{O}$ & $\mathrm{K}_{2} \mathrm{O}$ & $\mathrm{P}_{2} \mathrm{O}_{5}$ & $\mathrm{Fe}_{2} \mathrm{O}_{3} / \mathrm{FeO}$ & $\mathrm{Total} \mathrm{Fe}$ \\
\hline 1 & 65.68 & 0.75 & 17.80 & 3.91 & 2.51 & 0.29 & 2.86 & 0.64 & 2.15 & 3.26 & 0.16 & 1.56 & 6.70 \\
2 & 63.16 & 0.77 & 17.82 & 4.56 & 2.35 & 0.87 & 3.98 & 0.39 & 2.36 & 3.59 & 0.15 & 1.94 & 7.17 \\
3 & 65.46 & 0.81 & 17.99 & 4.36 & 2.48 & 0.19 & 2.89 & 0.74 & 1.45 & 3.48 & 0.16 & 1.76 & 7.11 \\
4 & 65.27 & 0.82 & 18.41 & 4.07 & 2.26 & 0.24 & 3.15 & 0.13 & 1.80 & 3.73 & 0.14 & 1.80 & 6.57 \\
5 & 65.70 & 0.78 & 17.61 & 4.70 & 2.28 & 0.14 & 2.87 & 0.64 & 1.74 & 3.38 & 0.16 & 2.06 & 7.24 \\
6 & 64.93 & 0.82 & 18.35 & 5.19 & 2.21 & 0.19 & 2.82 & 0.23 & 1.55 & 3.58 & 0.14 & 2.35 & 7.64 \\
7 & 64.41 & 0.77 & 17.63 & 5.29 & 1.70 & 0.69 & 2.43 & 0.78 & 2.79 & 3.33 & 0.17 & 3.11 & 7.18 \\
8 & 64.29 & 0.79 & 17.14 & 4.53 & 2.77 & 0.23 & 2.54 & 2.79 & 2.10 & 2.67 & 0.14 & 1.63 & 7.61 \\
9 & 64.39 & 0.79 & 18.51 & 5.21 & 2.00 & 0.25 & 2.82 & 0.70 & 1.57 & 3.61 & 0.15 & 2.61 & 7.43 \\
10 & 63.19 & 0.83 & 18.81 & 4.63 & 2.46 & 0.54 & 3.41 & 0.35 & 2.14 & 3.48 & 0.17 & 1.88 & 7.37 \\
11 & 63.73 & 0.78 & 18.37 & 4.08 & 3.17 & 0.22 & 2.95 & 1.13 & 1.94 & 3.45 & 0.18 & 1.29 & 7.60 \\
12 & 63.77 & 0.79 & 18.24 & 5.30 & 1.90 & 0.28 & 2.76 & 1.87 & 1.75 & 3.19 & 0.17 & 2.80 & 7.41 \\
13 & 63.42 & 0.79 & 18.15 & 5.20 & 2.02 & 0.23 & 2.93 & 1.75 & 2.12 & 3.24 & 0.15 & 2.58 & 7.45 \\
14 & 65.66 & 0.71 & 16.64 & 3.80 & 1.98 & 0.36 & 2.27 & 2.32 & 3.12 & 3.00 & 0.14 & 1.92 & 6.00 \\
15 & 66.52 & 0.69 & 17.21 & 4.00 & 1.75 & 0.17 & 2.34 & 1.96 & 1.99 & 3.25 & 0.13 & 2.29 & 5.94 \\
16 & 63.66 & 0.80 & 18.52 & 5.08 & 2.33 & 0.14 & 2.63 & 2.21 & 1.29 & 3.15 & 0.18 & 2.18 & 7.68 \\
17 & 65.49 & 0.69 & 15.99 & 3.62 & 2.88 & 0.16 & 2.35 & 4.10 & 2.18 & 2.37 & 0.16 & 1.26 & 6.82 \\
18 & 64.55 & 0.70 & 17.71 & 4.82 & 1.88 & 0.21 & 2.89 & 1.93 & 2.14 & 3.01 & 0.15 & 2.56 & 6.92 \\
19 & 62.83 & 0.76 & 18.26 & 5.31 & 1.90 & 0.36 & 3.25 & 1.93 & 1.87 & 3.35 & 0.17 & 2.79 & 7.42 \\
\hline
\end{tabular}

Tiba, T., 1974. Chemical composition of the deep sea core from the Philippine Sea. Bull. Nat. Sci. Museum, 17, 181-185.
Watts, A. B., and Weissel, J. K., 1975. Tectonic history of the Shikoku marginal basin. Earth Planet. Sci. Lett., 25, 239-250. 
TABLE 10

Chemical Composition (\%) Recalculated by Excluding Carbonates, Residual Materials, Water, and Salts, Hole 444A

\begin{tabular}{|c|c|c|c|c|c|c|c|c|c|c|c|c|c|}
\hline No. & $\mathrm{SiO}_{2}$ & $\mathrm{TiO}_{2}$ & $\mathrm{Al}_{2} \mathrm{O}_{3}$ & $\mathrm{Fe}_{2} \mathrm{O}_{3}$ & $\mathrm{FeO}$ & $\mathrm{MnO}$ & $\mathrm{MgO}$ & $\mathrm{CaO}$ & $\mathrm{Na}_{2} \mathrm{O}$ & $\mathrm{K}_{2} \mathrm{O}$ & $\mathrm{P}_{2} \mathrm{O}_{5}$ & $\mathrm{Fe}_{2} \mathrm{O}_{3} / \mathrm{FeO}$ & Total $\mathrm{Fe}$ \\
\hline $\begin{array}{l}1 \\
2 \\
3 \\
4 \\
5\end{array}$ & $\begin{array}{l}62.80 \\
63.07 \\
62.71 \\
63.54 \\
64.06\end{array}$ & $\begin{array}{l}0.77 \\
0.77 \\
0.84 \\
0.80 \\
0.73\end{array}$ & $\begin{array}{l}19.50 \\
17.92 \\
18.95 \\
18.74 \\
18.53\end{array}$ & $\begin{array}{l}5.36 \\
5.31 \\
6.38 \\
5.41 \\
5.18\end{array}$ & $\begin{array}{l}2.12 \\
2.20 \\
1.75 \\
2.01 \\
2.01\end{array}$ & $\begin{array}{l}0.26 \\
0.24 \\
0.50 \\
0.39 \\
0.23\end{array}$ & $\begin{array}{l}3.24 \\
2.84 \\
2.67 \\
3.01 \\
2.89\end{array}$ & $\begin{array}{l}0.65 \\
2.63 \\
0.15 \\
1.09 \\
1.62\end{array}$ & $\begin{array}{l}1.78 \\
2.07 \\
1.71 \\
1.43 \\
1.56\end{array}$ & $\begin{array}{l}3.34 \\
2.79 \\
4.03 \\
3.38 \\
3.02\end{array}$ & $\begin{array}{l}0.19 \\
0.17 \\
0.32 \\
0.19 \\
0.16\end{array}$ & $\begin{array}{l}2.52 \\
2.41 \\
3.65 \\
2.69 \\
2.58\end{array}$ & $\begin{array}{l}7.72 \\
7.75 \\
8.32 \\
7.65 \\
7.42\end{array}$ \\
\hline $\begin{array}{r}6 \\
7 \\
8 \\
9 \\
10\end{array}$ & $\begin{array}{l}63.27 \\
71.20 \\
65.59 \\
61.99 \\
61.83\end{array}$ & $\begin{array}{l}0.73 \\
0.55 \\
0.65 \\
0.75 \\
0.79\end{array}$ & $\begin{array}{l}17.99 \\
14.51 \\
17.51 \\
17.90 \\
18.35\end{array}$ & $\begin{array}{l}5.43 \\
3.19 \\
4.43 \\
5.42 \\
5.66\end{array}$ & $\begin{array}{l}1.90 \\
1.79 \\
2.13 \\
2.19 \\
1.81\end{array}$ & $\begin{array}{l}0.32 \\
0.17 \\
0.12 \\
0.37 \\
0.39\end{array}$ & $\begin{array}{l}2.68 \\
1.52 \\
2.55 \\
2.79 \\
3.22\end{array}$ & $\begin{array}{l}2.38 \\
2.39 \\
2.15 \\
2.96 \\
2.77\end{array}$ & $\begin{array}{l}2.06 \\
2.41 \\
1.53 \\
2.65 \\
2.20\end{array}$ & $\begin{array}{l}3.05 \\
2.18 \\
3.22 \\
2.78 \\
2.80\end{array}$ & $\begin{array}{l}0.18 \\
0.097 \\
0.13 \\
0.20 \\
0.19\end{array}$ & $\begin{array}{l}2.85 \\
1.78 \\
2.07 \\
2.47 \\
3.13\end{array}$ & $\begin{array}{l}7.54 \\
5.17 \\
6.80 \\
7.85 \\
7.67\end{array}$ \\
\hline $\begin{array}{l}11 \\
12 \\
13 \\
14 \\
15\end{array}$ & $\begin{array}{l}61.56 \\
62.36 \\
62.58 \\
62.42 \\
61.99\end{array}$ & $\begin{array}{l}0.82 \\
0.80 \\
0.77 \\
0.70 \\
0.73\end{array}$ & $\begin{array}{l}18.03 \\
18.62 \\
17.86 \\
18.53 \\
19.06\end{array}$ & $\begin{array}{l}5.30 \\
5.98 \\
5.48 \\
6.42 \\
6.49\end{array}$ & $\begin{array}{l}2.45 \\
1.92 \\
1.82 \\
1.12 \\
1.25\end{array}$ & $\begin{array}{l}0.37 \\
0.47 \\
0.42 \\
0.39 \\
0.39\end{array}$ & $\begin{array}{l}3.19 \\
3.33 \\
3.01 \\
3.18 \\
2.86\end{array}$ & $\begin{array}{l}3.10 \\
1.32 \\
2.63 \\
2.12 \\
2.08\end{array}$ & $\begin{array}{l}2.39 \\
2.05 \\
2.46 \\
1.97 \\
1.94\end{array}$ & $\begin{array}{l}2.58 \\
2.94 \\
2.78 \\
2.95 \\
2.99\end{array}$ & $\begin{array}{l}0.21 \\
0.20 \\
0.19 \\
0.20 \\
0.21\end{array}$ & $\begin{array}{l}2.17 \\
3.11 \\
3.02 \\
5.71 \\
5.20\end{array}$ & $\begin{array}{l}8.02 \\
8.11 \\
7.50 \\
7.67 \\
7.88\end{array}$ \\
\hline $\begin{array}{l}16 \\
17 \\
18 \\
19 \\
20\end{array}$ & $\begin{array}{l}61.52 \\
63.09 \\
62.28 \\
61.50 \\
62.13\end{array}$ & $\begin{array}{l}0.77 \\
0.74 \\
0.73 \\
0.77 \\
0.78\end{array}$ & $\begin{array}{l}18.29 \\
18.48 \\
18.72 \\
18.40 \\
18.69\end{array}$ & $\begin{array}{l}7.06 \\
5.93 \\
6.06 \\
6.80 \\
6.82\end{array}$ & $\begin{array}{l}1.25 \\
1.10 \\
1.40 \\
1.21 \\
1.30\end{array}$ & $\begin{array}{l}0.55 \\
0.42 \\
0.35 \\
0.45 \\
0.33\end{array}$ & $\begin{array}{l}3.13 \\
2.84 \\
2.93 \\
3.34 \\
3.29\end{array}$ & $\begin{array}{l}2.09 \\
1.65 \\
2.33 \\
2.29 \\
1.86\end{array}$ & $\begin{array}{l}2.17 \\
2.35 \\
2.15 \\
2.10 \\
1.82\end{array}$ & $\begin{array}{l}3.01 \\
3.20 \\
2.89 \\
2.96 \\
2.82\end{array}$ & $\begin{array}{l}0.16 \\
0.20 \\
0.17 \\
0.19 \\
0.15\end{array}$ & $\begin{array}{l}5.67 \\
5.40 \\
4.34 \\
5.60 \\
5.24\end{array}$ & $\begin{array}{l}8.45 \\
7.15 \\
7.61 \\
8.15 \\
8.27\end{array}$ \\
\hline $\begin{array}{l}21 \\
22 \\
23 \\
24 \\
25\end{array}$ & $\begin{array}{l}63.24 \\
59.10 \\
59.66 \\
61.48 \\
59.99\end{array}$ & $\begin{array}{l}0.75 \\
0.77 \\
0.78 \\
0.81 \\
0.79\end{array}$ & $\begin{array}{l}17.77 \\
16.75 \\
16.54 \\
16.57 \\
16.61\end{array}$ & $\begin{array}{l}6.56 \\
6.17 \\
6.25 \\
5.61 \\
5.76\end{array}$ & $\begin{array}{l}1.99 \\
2.77 \\
2.93 \\
3.42 \\
3.62\end{array}$ & $\begin{array}{l}0.48 \\
0.61 \\
0.58 \\
0.33 \\
0.28\end{array}$ & $\begin{array}{l}2.89 \\
3.81 \\
3.95 \\
3.41 \\
3.57\end{array}$ & $\begin{array}{l}3.67 \\
4.84 \\
4.47 \\
3.49 \\
4.95\end{array}$ & $\begin{array}{l}0.14 \\
3.08 \\
2.69 \\
2.52 \\
2.40\end{array}$ & $\begin{array}{l}2.36 \\
1.92 \\
2.00 \\
1.77 \\
1.88\end{array}$ & $\begin{array}{l}0.16 \\
0.17 \\
0.15 \\
0.59 \\
0.15\end{array}$ & $\begin{array}{l}3.29 \\
2.23 \\
2.14 \\
1.64 \\
1.59\end{array}$ & $\begin{array}{l}8.78 \\
9.24 \\
9.51 \\
9.41 \\
9.78\end{array}$ \\
\hline $\begin{array}{l}26 \\
27 \\
28 \\
29 \\
30\end{array}$ & $\begin{array}{l}59.83 \\
64.93 \\
71.98 \\
64.77 \\
73.32\end{array}$ & $\begin{array}{l}0.87 \\
0.76 \\
0.46 \\
0.69 \\
0.49\end{array}$ & $\begin{array}{l}16.02 \\
15.76 \\
14.12 \\
16.77 \\
14.10\end{array}$ & $\begin{array}{l}7.12 \\
4.15 \\
2.12 \\
4.12 \\
2.55\end{array}$ & $\begin{array}{l}3.83 \\
3.41 \\
1.77 \\
2.60 \\
1.97\end{array}$ & $\begin{array}{l}0.25 \\
0.22 \\
0.15 \\
0.21 \\
0.19\end{array}$ & $\begin{array}{l}3.61 \\
2.47 \\
1.58 \\
2.39 \\
1.59\end{array}$ & $\begin{array}{l}5.31 \\
4.10 \\
2.83 \\
4.11 \\
0.96\end{array}$ & $\begin{array}{l}1.49 \\
2.59 \\
2.65 \\
2.20 \\
2.61\end{array}$ & $\begin{array}{l}1.54 \\
1.45 \\
2.23 \\
2.00 \\
2.12\end{array}$ & $\begin{array}{l}0.14 \\
0.15 \\
0.11 \\
0.15 \\
0.10\end{array}$ & $\begin{array}{l}1.86 \\
1.22 \\
1.20 \\
1.59 \\
1.29\end{array}$ & $\begin{array}{r}11.37 \\
7.94 \\
4.10 \\
7.00 \\
4.75\end{array}$ \\
\hline $\begin{array}{l}31 \\
32 \\
33 \\
34 \\
35\end{array}$ & $\begin{array}{l}63.84 \\
62.32 \\
60.73 \\
59.31 \\
60.80\end{array}$ & $\begin{array}{l}0.65 \\
0.84 \\
0.77 \\
0.80 \\
0.80\end{array}$ & $\begin{array}{l}16.64 \\
17.56 \\
16.53 \\
16.57 \\
17.23\end{array}$ & $\begin{array}{l}4.54 \\
5.72 \\
6.35 \\
6.27 \\
6.26\end{array}$ & $\begin{array}{l}3.14 \\
3.72 \\
2.83 \\
3.75 \\
3.12\end{array}$ & $\begin{array}{l}0.25 \\
0.35 \\
0.20 \\
0.18 \\
0.34\end{array}$ & $\begin{array}{l}2.79 \\
3.73 \\
3.52 \\
3.64 \\
3.21\end{array}$ & $\begin{array}{l}3.85 \\
0.75 \\
4.76 \\
6.19 \\
4.34\end{array}$ & $\begin{array}{l}1.89 \\
2.25 \\
2.08 \\
1.63 \\
1.58\end{array}$ & $\begin{array}{l}2.24 \\
2.59 \\
2.06 \\
1.52 \\
2.17\end{array}$ & $\begin{array}{l}0.16 \\
0.16 \\
0.16 \\
0.14 \\
0.14\end{array}$ & $\begin{array}{l}1.44 \\
1.54 \\
2.24 \\
1.67 \\
2.01\end{array}$ & $\begin{array}{r}8.03 \\
9.86 \\
9.50 \\
10.44 \\
9.73\end{array}$ \\
\hline $\begin{array}{l}36 \\
37 \\
38 \\
39 \\
40\end{array}$ & $\begin{array}{l}61.83 \\
63.83 \\
68.60 \\
75.38 \\
62.83\end{array}$ & $\begin{array}{l}0.77 \\
0.75 \\
0.64 \\
0.49 \\
0.73\end{array}$ & $\begin{array}{l}16.76 \\
16.83 \\
14.54 \\
12.56 \\
17.44\end{array}$ & $\begin{array}{l}6.14 \\
5.10 \\
2.81 \\
1.21 \\
5.64\end{array}$ & $\begin{array}{l}3.07 \\
3.09 \\
2.99 \\
2.11 \\
2.19\end{array}$ & $\begin{array}{l}0.18 \\
0.27 \\
0.15 \\
0.086 \\
0.23\end{array}$ & $\begin{array}{l}3.29 \\
3.17 \\
2.13 \\
0.69 \\
3.05\end{array}$ & $\begin{array}{l}3.71 \\
2.65 \\
3.81 \\
2.80 \\
3.13\end{array}$ & $\begin{array}{l}1.69 \\
1.85 \\
2.50 \\
2.70 \\
1.92\end{array}$ & $\begin{array}{l}2.40 \\
2.30 \\
1.68 \\
1.89 \\
2.65\end{array}$ & $\begin{array}{l}0.14 \\
0.17 \\
0.14 \\
0.090 \\
0.18\end{array}$ & $\begin{array}{l}2.00 \\
1.65 \\
0.94 \\
0.57 \\
2.57\end{array}$ & $\begin{array}{l}9.56 \\
8.53 \\
6.14 \\
3.55 \\
8.07\end{array}$ \\
\hline $\begin{array}{l}41 \\
42 \\
43 \\
44 \\
45\end{array}$ & $\begin{array}{l}62.23 \\
73.52 \\
66.23 \\
61.22 \\
69.13\end{array}$ & $\begin{array}{l}0.69 \\
0.44 \\
0.67 \\
0.71 \\
0.56\end{array}$ & $\begin{array}{l}17.39 \\
13.21 \\
16.33 \\
17.37 \\
14.13\end{array}$ & $\begin{array}{l}4.96 \\
1.90 \\
4.79 \\
6.43 \\
3.29\end{array}$ & $\begin{array}{l}2.43 \\
1.87 \\
2.27 \\
2.70 \\
1.66\end{array}$ & $\begin{array}{l}0.28 \\
0.13 \\
0.17 \\
0.11 \\
0.17\end{array}$ & $\begin{array}{l}3.01 \\
1.19 \\
2.68 \\
3.65 \\
1.91\end{array}$ & $\begin{array}{l}4.38 \\
2.86 \\
2.92 \\
3.57 \\
3.98\end{array}$ & $\begin{array}{l}2.38 \\
2.57 \\
1.55 \\
1.47 \\
1.93\end{array}$ & $\begin{array}{l}2.07 \\
2.22 \\
2.24 \\
2.63 \\
3.14\end{array}$ & $\begin{array}{l}0.19 \\
0.091 \\
0.14 \\
0.13 \\
0.11\end{array}$ & $\begin{array}{l}2.04 \\
1.01 \\
2.11 \\
2.38 \\
1.98\end{array}$ & $\begin{array}{l}7.65 \\
3.98 \\
7.32 \\
9.43 \\
5.13\end{array}$ \\
\hline $\begin{array}{l}46 \\
47 \\
48 \\
49 \\
50\end{array}$ & $\begin{array}{l}66.52 \\
61.82 \\
63.11 \\
64.62 \\
67.18\end{array}$ & $\begin{array}{l}0.54 \\
0.70 \\
0.69 \\
0.66 \\
0.67\end{array}$ & $\begin{array}{l}15.98 \\
15.79 \\
15.80 \\
15.55 \\
14.52\end{array}$ & $\begin{array}{l}4.21 \\
6.32 \\
5.60 \\
4.85 \\
2.93\end{array}$ & $\begin{array}{l}2.26 \\
2.50 \\
2.70 \\
2.51 \\
3.16\end{array}$ & $\begin{array}{l}0.11 \\
0.23 \\
0.23 \\
0.23 \\
0.22\end{array}$ & $\begin{array}{l}3.13 \\
4.14 \\
3.44 \\
3.19 \\
2.19\end{array}$ & $\begin{array}{l}2.17 \\
3.92 \\
3.93 \\
3.88 \\
3.43\end{array}$ & $\begin{array}{l}1.41 \\
1.94 \\
1.95 \\
1.93 \\
3.06\end{array}$ & $\begin{array}{l}3.53 \\
2.45 \\
2.36 \\
2.38 \\
2.53\end{array}$ & $\begin{array}{l}0.13 \\
0.18 \\
0.20 \\
0.20 \\
0.13\end{array}$ & $\begin{array}{l}1.86 \\
2.53 \\
2.08 \\
1.93 \\
0.93\end{array}$ & $\begin{array}{l}6.73 \\
9.09 \\
8.60 \\
7.64 \\
6.43\end{array}$ \\
\hline $\begin{array}{l}51 \\
52 \\
53 \\
54 \\
55\end{array}$ & $\begin{array}{l}68.06 \\
62.54 \\
60.89 \\
61.54 \\
64.88\end{array}$ & $\begin{array}{l}0.57 \\
0.70 \\
0.71 \\
0.71 \\
0.68\end{array}$ & $\begin{array}{l}15.30 \\
15.70 \\
15.02 \\
15.78 \\
15.26\end{array}$ & $\begin{array}{l}3.87 \\
7.19 \\
7.43 \\
6.53 \\
6.62\end{array}$ & $\begin{array}{l}2.25 \\
1.80 \\
1.97 \\
2.10 \\
1.89\end{array}$ & $\begin{array}{l}0.37 \\
0.43 \\
1.29 \\
0.62 \\
0.73\end{array}$ & $\begin{array}{l}2.51 \\
4.42 \\
5.73 \\
4.87 \\
2.65\end{array}$ & $\begin{array}{l}1.71 \\
2.02 \\
0.36 \\
2.55 \\
0.29\end{array}$ & $\begin{array}{l}2.34 \\
1.99 \\
3.60 \\
2.22 \\
3.71\end{array}$ & $\begin{array}{l}2.89 \\
3.09 \\
2.86 \\
2.95 \\
3.01\end{array}$ & $\begin{array}{l}0.12 \\
0.12 \\
0.13 \\
0.13 \\
0.27\end{array}$ & $\begin{array}{l}1.72 \\
4.00 \\
3.78 \\
3.11 \\
3.51\end{array}$ & $\begin{array}{l}6.37 \\
9.19 \\
9.62 \\
8.87 \\
8.72\end{array}$ \\
\hline
\end{tabular}


TABLE 11

Average Chemical Compositions (\%) and Standard Deviations of Sediments from Leg 58 Sites and Other Areas

\begin{tabular}{|c|c|c|c|c|c|c|c|c|c|c|}
\hline & Site 442 & Site 443 & Site 444 & $\begin{array}{l}\text { Nankai } \\
\text { Trough }\end{array}$ & $\underset{\text { Trenchb }}{\text { Japan }}$ & $\begin{array}{c}\text { Yamato Bank } \\
\text { Areac }\end{array}$ & $\begin{array}{c}\text { Nishitsugaru } \\
\text { Basind }\end{array}$ & $\begin{array}{l}\text { Around the } \\
\text { Oki Islandse }\end{array}$ & $\begin{array}{l}\text { South } \\
\text { Philippine } \\
\text { Seaf }\end{array}$ & $\begin{array}{l}\text { East Pacific } \\
\text { Ocean Pelagic } \\
\text { Sedimentss }\end{array}$ \\
\hline $\begin{array}{l}\text { Number } \\
\text { of Samples }\end{array}$ & 91 & 114 & 74 & 29 & 82 & 69 & 87 & 45 & 15 & 35 \\
\hline $\begin{array}{l}\mathrm{SiO}_{2} \\
\mathrm{TiO}_{2} \\
\mathrm{Al}_{2} \mathrm{O}_{3}\end{array}$ & $\begin{aligned} 63.44 & \pm 3.27 \\
0.74 & \pm 0.08 \\
17.77 & \pm 1.53\end{aligned}$ & $\begin{aligned} 64.07 & \pm 2.54 \\
0.74 & \pm 0.07 \\
17.57 & \pm 1.06\end{aligned}$ & $\begin{aligned} 64.04 & \pm 3.22 \\
0.73 & \pm 0.09 \\
17.03 & \pm 1.52\end{aligned}$ & $\begin{aligned} 66.68 & \pm 1.99 \\
0.72 & \pm 0.06 \\
16.51 & \pm 0.64\end{aligned}$ & $\begin{aligned} 68.84 & \pm 2.78 \\
0.62 & \pm 0.07 \\
13.60 & \pm 1.47\end{aligned}$ & $\begin{aligned} 65.80 & \pm 3.84 \\
0.73 & \pm 0.11 \\
16.54 & \pm 1.96\end{aligned}$ & $\begin{aligned} 66.58 & \pm 2.02 \\
0.67 & \pm 0.05 \\
16.12 & \pm 0.63\end{aligned}$ & $\begin{aligned} 75.59 & \pm 2.57 \\
0.50 & \pm 0.082 \\
12.81 & \pm 1.39\end{aligned}$ & $\begin{array}{r}54.78 \pm 0.59 \\
1.05 \pm 0.10 \\
21.54 \pm 0.86\end{array}$ & $\begin{array}{r}49.84 \\
1.22 \\
17.38\end{array}$ \\
\hline $\begin{array}{l}\text { Total Fe } \\
\text { as } \mathrm{Fe}_{2} \mathrm{O}_{3}\end{array}$ & $7.57 \pm 1.20$ & $7.51 \pm 1.20$ & $7.68 \pm 1.45$ & $6.01 \pm 0.65$ & $5.67 \pm 0.92$ & $6.94 \pm 1.54$ & $5.85 \pm 0.45$ & $4.03 \pm 0.81$ & $11.45 \pm 1.34$ & 9.29 \\
\hline $\begin{array}{l}\mathrm{MnO} \\
\mathrm{MgO} \\
\mathrm{CaO} \\
\mathrm{Na}_{2} \mathrm{O} \\
\mathrm{K}_{2} \mathrm{O} \\
\mathrm{P}_{2} \mathrm{O}_{5}\end{array}$ & $\begin{array}{l}0.65 \pm 0.73 \\
3.13 \pm 0.92 \\
1.28 \pm 0.69 \\
2.13 \pm 1.04 \\
3.32 \pm 0.38 \\
0.18 \pm 0.14\end{array}$ & $\begin{array}{l}0.50 \pm 0.49 \\
2.96 \pm 0.52 \\
1.40 \pm 1.78 \\
2.07 \pm 0.69 \\
3.26 \pm 0.44 \\
0.18 \pm 0.23\end{array}$ & $\begin{array}{l}0.32 \pm 0.20 \\
2.97 \pm 0.76 \\
2.48 \pm 1.42 \\
2.10 \pm 0.54 \\
2.74 \pm 0.59 \\
0.17 \pm 0.06\end{array}$ & $\begin{array}{l}0.09 \pm 0.06 \\
2.29 \pm 0.36 \\
1.51 \pm 0.93 \\
3.29 \pm 0.36 \\
3.09 \pm 0.41 \\
0.14 \pm 0.01\end{array}$ & $\begin{array}{l}0.07 \pm 0.02 \\
3.11 \pm 0.37 \\
2.38 \pm 1.19 \\
3.77 \pm 0.49 \\
2.12 \pm 0.30 \\
0.15 \pm 0.13\end{array}$ & $\begin{array}{l}0.48 \pm 1.95 \\
2.91 \pm 0.71 \\
1.04 \pm 0.65 \\
2.16 \pm 0.75 \\
3.47 \pm 0.47 \\
0.16 \pm 0.075\end{array}$ & $\begin{array}{l}0.07 \pm 0.04 \\
3.31 \pm 0.42 \\
1.93 \pm 0.90 \\
3.00 \pm 0.54 \\
2.49 \pm 0.28 \\
0.17 \pm 0.15\end{array}$ & $\begin{aligned} 0.044 & \pm 0.013 \\
1.16 & \pm 0.34 \\
1.57 & \pm 0.53 \\
1.28 & \pm 0.18 \\
2.99 & \pm 0.25 \\
0.097 & \pm 0.021\end{aligned}$ & $\begin{array}{l}0.68 \pm 0.27 \\
3.45 \pm 0.26 \\
1.30 \pm 0.15 \\
2.51 \pm 0.45 \\
2.93 \pm 0.16 \\
0.37 \pm 0.15\end{array}$ & $\begin{array}{l}1.98 \\
3.48 \\
4.06 \\
5.39 \\
3.01\end{array}$ \\
\hline
\end{tabular}

${ }^{\text {a S}}$ Sugisaki (1978); this area comprises the continental shelf and slope of the Pacific Side of southwest Japan, the Nankai Trough, and the northern Shikoku Basin. Sugisaki and Honza (in press).

c Sugisaki (in press b).

Sugisaki (in press a).

e Sugisaki and Kinoshita (in press),

Tiba (1974); core at Lat. $14^{\circ} 09^{\prime} \mathrm{N}$, Long. $130^{\circ} 04^{\prime} \mathrm{E}$.

$\mathrm{g}_{\mathrm{Goldberg}}$ and Arrhenius (1958). 

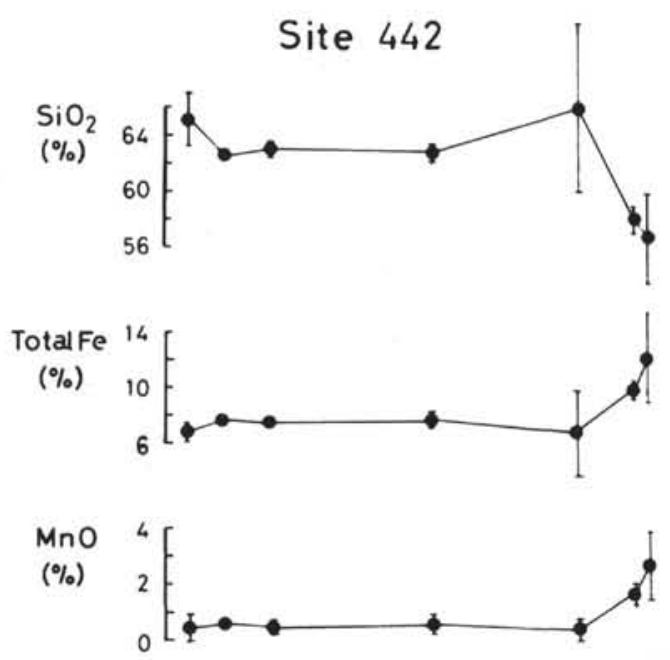

Site 443

$\mathrm{SiO}_{2}$

$(\%)$
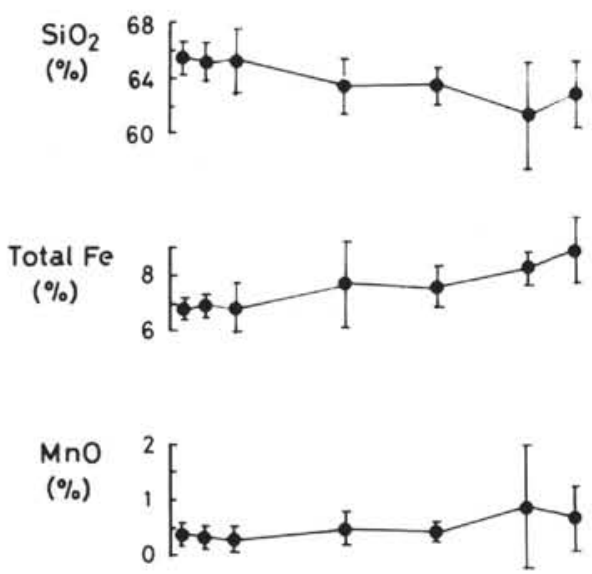

Site 444
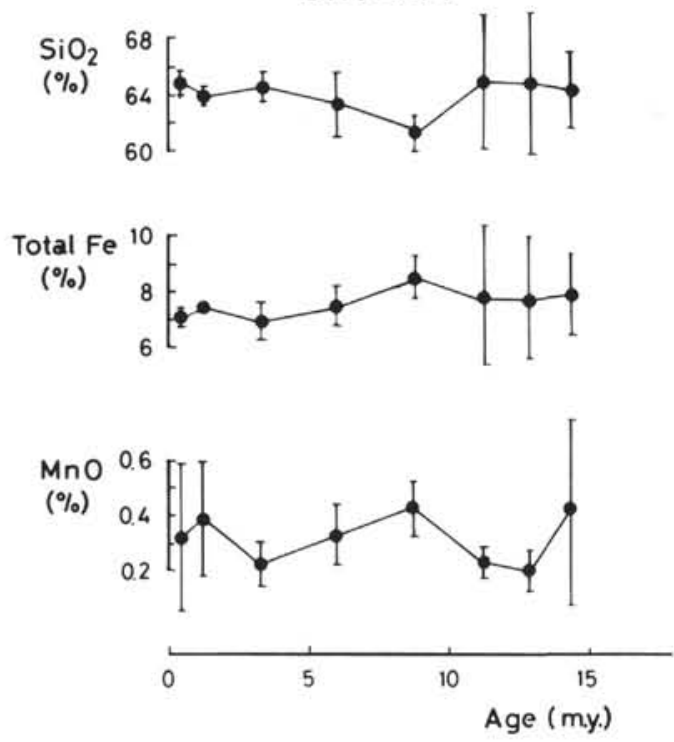

Figure 1. Temporal variation of $\mathrm{SiO}_{2}$, total $\mathrm{Fe}$ as $\mathrm{Fe}_{2} \mathrm{O}_{3}$, and $\mathrm{MnO}$ at the Shikoku Basin sites. Each point represents an average value for each lithologic unit of the cored sediments (see respective site chapters, this volume). The error bar shows standard deviation.

\section{TABLE 12}

Chemical Compositions (\%) of Manganese-Rich Sediments (silicate composition excluding carbonate, water, etc.)

\begin{tabular}{|c|c|c|}
\hline & Lithologic Unit V, Site $442^{\mathrm{a}}$ & Red Shaleb \\
\hline $\mathrm{SiO}_{2}$ & $56.83 \pm 3.18$ & 60.38 \\
\hline $\mathrm{TiO}_{2}$ & $0.56 \pm 0.083$ & 0.73 \\
\hline $\mathrm{Al}_{2} \mathrm{O}_{3}$ & $13.67 \pm 2.42$ & 17.13 \\
\hline Total Fe & & \\
\hline $\begin{array}{l}\underset{\mathrm{MnO}}{\operatorname{ar}} \mathrm{Fe}_{2} \mathrm{O}_{3} \\
\end{array}$ & $\begin{aligned} 12.21 & \pm 3.26 \\
2.67 & \pm 1.30\end{aligned}$ & $\begin{array}{r}12.65 \\
1.18\end{array}$ \\
\hline $\mathrm{MgO}$ & $5.15 \pm 1.14$ & 1.01 \\
\hline $\mathrm{CaO}$ & $1.33 \pm 0.18$ & 0.55 \\
\hline $\mathrm{Na}_{2} \mathrm{O}$ & $4.48 \pm 1.50$ & 1.95 \\
\hline $\mathrm{K}_{2} \mathrm{O}$ & $2.91 \pm 0.35$ & 4.82 \\
\hline $\mathrm{P}_{2} \mathrm{O}_{5}$ & $0.20 \pm 0.04$ & 0.18 \\
\hline
\end{tabular}

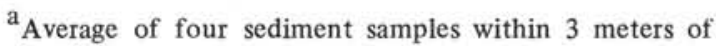
basalt basement.

${ }^{b}$ Red shale above pillow basalt body in the Shimanto Belt in Kyushu, Cretaceous geosynclinal area, southwest Japan; Sugisaki et al. (1979) describe the chemical composition of the basalt. 

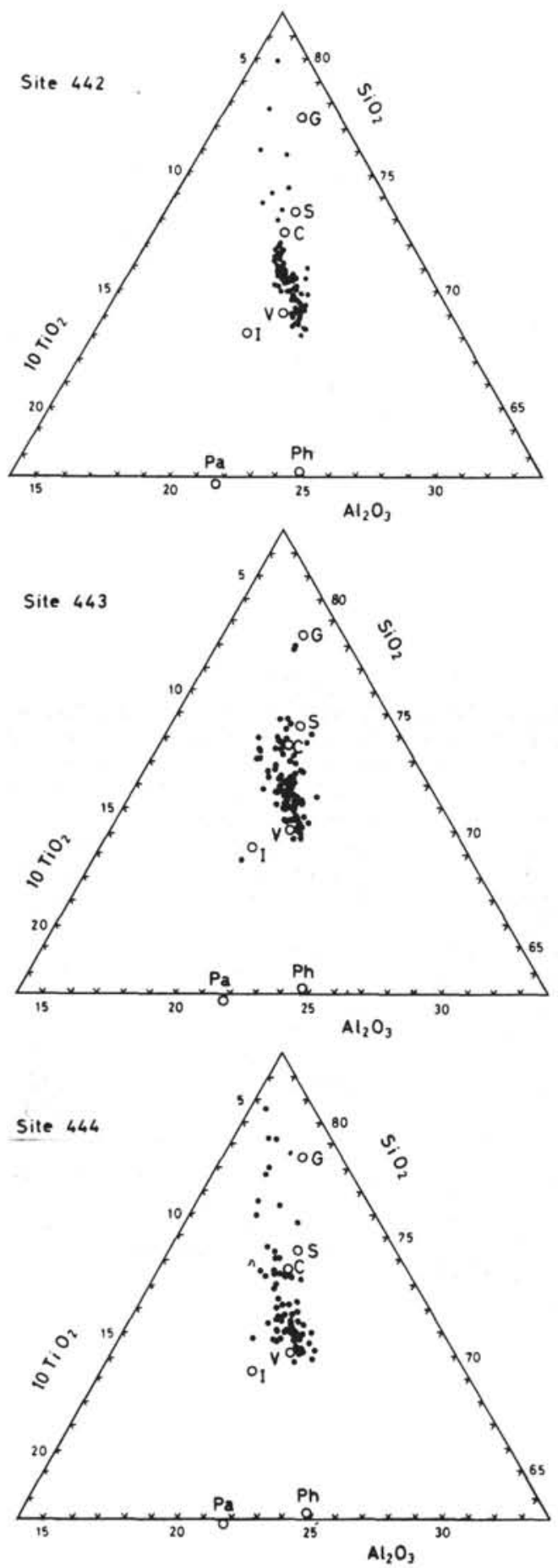

Figure 2. Relationships among $\mathrm{SiO}_{2}, \mathrm{TiO}_{2}$ and $\mathrm{Al}_{2} \mathrm{O}_{3}$. Closed circles represent argillaceous sediments analyzed. Open circles represent averaged rocks: G, Japanese granites (Geological Survey of Japan, 1960); V, Quaternary volcanics (Sugisaki, 1976); C, shales from the Paleozoic Chichibu terrain, Japan (Ono, 1976); S, shales from the Mesozoic Shimanto terrain, Japan (Ono, 1976); I, Quaternary volcanics from the Izu-Mariana Islands (Sugisaki, 1976); Ph, average sediments in a core from the Philippine Sea (Lat. $14^{\circ} 09^{\prime \prime} \mathrm{N}$, Long. $130^{\circ} 04^{\prime \prime} \mathrm{E}$; Tiba, 1974); $\mathrm{Pa}$, sediments from the east Pacific Ocean (Goldberg and Arrhenius, 1958).
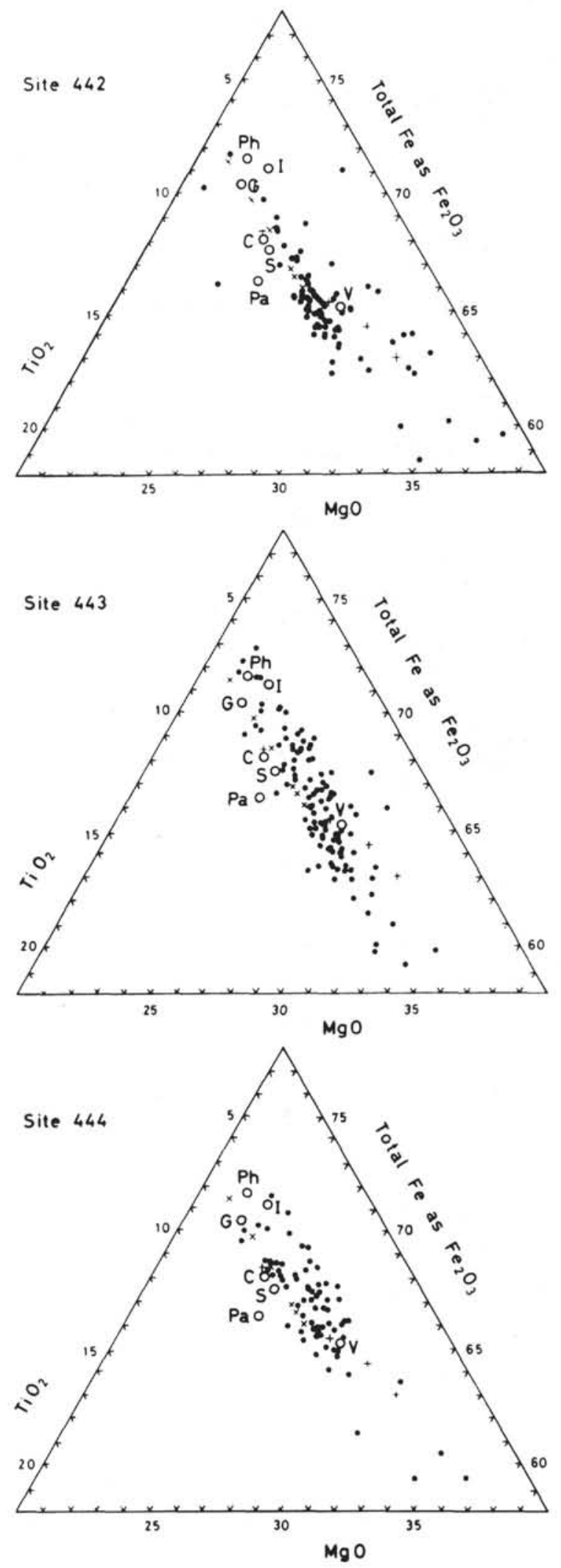

Figure 3. Relationships among $\mathrm{TiO}_{2}$, total $\mathrm{Fe}$ as $\mathrm{Fe}_{2} \mathrm{O}_{3}$, and $\mathrm{MgO}$. Crosses and Xs represent averaged variation trends of Japanese volcanics (Sugisaki, 1976) and Japanese granites (Aramaki et al., 1972), respectively. Other symbols are the same as those in Figure 2. 


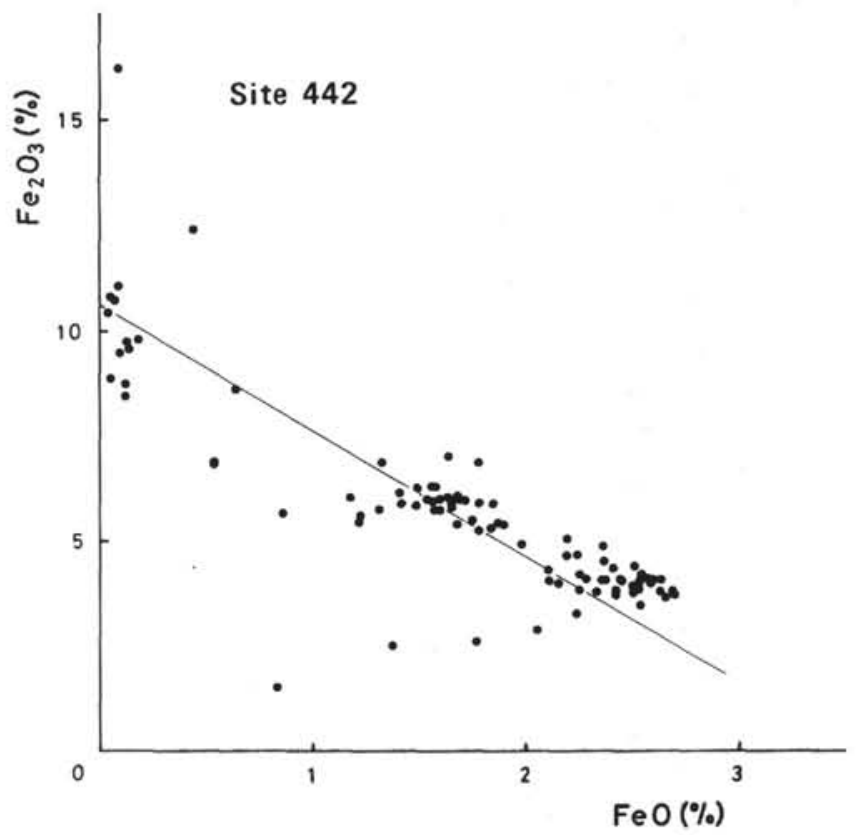

Figure 4. Relation between $\mathrm{FeO}$ and $\mathrm{Fe}_{2} \mathrm{O}_{3}$ at Site 442 .

The regression line is $\mathrm{Fe}_{2} \mathrm{O}_{3}=-2.93 \mathrm{FeO}+10.62$, with a correlation coefficient of -0.832 . 